\title{
Total Synthesis of $(-)$-Goniomitine
}

\author{
Shiqiang Zhou, ${ }^{\dagger}$ Yanxing Jia*, ${ }^{\dagger, \dagger}$ \\ †State Key Laboratory of Natural and Biomimetic Drugs, School of Pharmaceutical Sciences, \\ Peking University, 38 Xueyuan Road, Beijing 100191, China; \\ "State Key Laboratory of Applied Organic Chemistry, Lanzhou University, Lanzhou 730000, \\ China.
}

yxjia@bjmu.edu.cn

\section{Table of Contents}

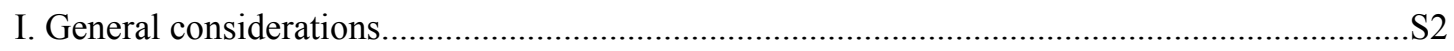

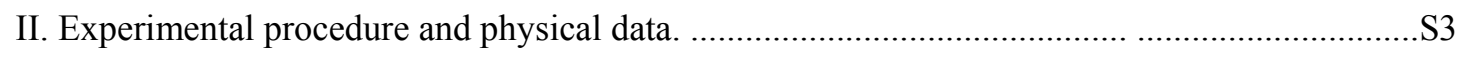

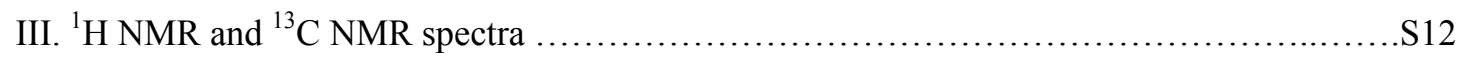




\section{General considerations}

Unless otherwise noted, all experiments were carried out under air atmosphere. Dichloromethane (DCM) was distilled over $\mathrm{CaH}_{2}$, and tetrahydrofuran (THF) were distilled over Na-Kalloy. The other reagents and solvents were directly used from the supplier without further purification unless noted. Analytical thin-layer chromatography (TLC) was carried out using 0.2 mm commercial silica gel plates (silica gel 60, F254, Merck). Visualization was achieved under a UV lamp (254 nm and $365 \mathrm{~nm}$ ), and by developing the plates with phosphomolybdic acid in ethanol. Flash chromatography was performed using silica gel (200-300 mesh) with solvents distilled prior to use.

Proton NMR $\left({ }^{1} \mathrm{H}\right)$ were recorded at $400 \mathrm{MHz}$ NMR spectrometer, Carbon NMR $\left({ }^{13} \mathrm{C}\right)$ at 100 $\mathrm{MHz}$ NMR spectrometer unless otherwise stated. For $\mathrm{CDCl}_{3}$ solutions the chemical shifts are reported as parts per million ( $\mathrm{ppm}$ ) referenced to residual protium or carbon of the solvents; $\mathrm{CHCl}_{3} \delta \mathrm{H}(7.26 \mathrm{ppm})$, TMS $\delta \mathrm{H}(0.00 \mathrm{ppm})$ and $\mathrm{CDCl}_{3} \delta \mathrm{C}(77.00 \mathrm{ppm})$. Coupling constants (J) are reported in Hertz $(\mathrm{Hz})$. Data for ${ }^{1} \mathrm{H}$ NMR spectra are reported as follows: chemical shift (ppm, referenced to protium; $\mathrm{s}=$ singlet, $\mathrm{d}=$ doublet, $\mathrm{t}=$ triplet, $\mathrm{q}=$ quartet, $\mathrm{p}=$ pentet (quintet), sept $=$ septet, $\mathrm{dd}=$ doublet of doublets, $\mathrm{td}=$ triplet of doublets, $\mathrm{ddd}=$ doublet of doublet of doublets, $\mathrm{m}=$ multiplet, br $\mathrm{s}=$ broad singlet), coupling constant $(\mathrm{Hz})$, and integration.

Infrared spectra were recorded with a thin layer of the product on a $\mathrm{KBr}$ disk and reported in frequency of absorption $\left(\mathrm{cm}^{-1}\right)$.

High resolution mass spectral (HRMS) data were obtained with an ionization mode of ESI.

The following abbreviations are used: FCC: flash column chromatography; PE: petroleum ether; EtOAc: ethyl acetate; DCM: dichloromethane; MeOH: methanol; THF: tetrahydrofuran; DMF: N, N-dimethylformamide; 


\section{Experimental procedure and physical data}

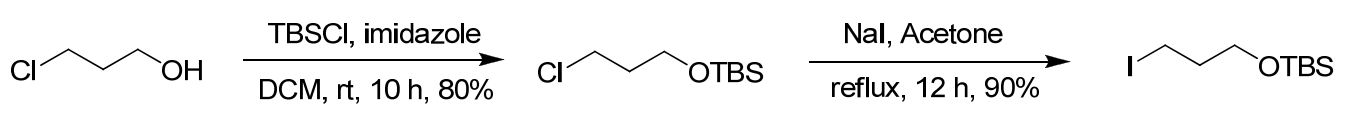

Compound 12 ${ }^{1}$ : To a solution of 3-chloro-propan-1-ol $(5.1 \mathrm{~mL}, 60.0 \mathrm{mmol})$ in DCM (120 mL) was added imidazole $(5.3 \mathrm{~g}, 78 \mathrm{mmol})$. After $5 \mathrm{~min}$ of stirring, tert-butyldimethylsilyl chloride (10.85 g, $72 \mathrm{mmol}$ ) was added over $5 \mathrm{~min}$. The reaction mixture was stirred for $10 \mathrm{~h}$ before being diluted with DCM $(50 \mathrm{~mL})$ and washed with saturated aqueous $\mathrm{NH}_{4} \mathrm{Cl}(150 \mathrm{~mL})$. The aqueous phase was extracted with DCM $(100 \mathrm{~mL} \times 3)$. The combined organic phase was washed with brine, dried over anhydrous $\mathrm{Na}_{2} \mathrm{SO}_{4}$, and reduced to dryness. Purification of the residue by FCC (PE : EtOAc $=20: 1)$ afforded the 3-chloro-1-tert-butyldimethylsiloxypropane $(10.0 \mathrm{~g}, 80 \%)$ as colorless liquid. To the solution of 3-chloro-1-tert-butyldimethylsiloxypropane (5 g, $24.0 \mathrm{mmol})$ in acetone $(120 \mathrm{~mL})$ was added $\mathrm{NaI}(36 \mathrm{~g}, 240 \mathrm{mmol})$ and the solution was refluxed for $12 \mathrm{~h}$. After cooling, the reaction mixture was evaporated and diluted with EtOAc $(150 \mathrm{~mL})$, washed with brine $(100$ $\mathrm{mL}$ ) and dried over anhydrous $\mathrm{Na}_{2} \mathrm{SO}_{4}$. After the solution was reduced to dryness, the residue was purified by FCC (PE:EtOAc $=20: 1)$ to yield 12 as colorless oil (6.5 g, 90\%). ${ }^{1} \mathrm{H}$ NMR (400 MHz, $\left.\mathrm{CDCl}_{3}\right) \delta 3.67(\mathrm{t}, J=5.6 \mathrm{~Hz}, 2 \mathrm{H}), 3.28(\mathrm{t}, J=6.8 \mathrm{~Hz}, 2 \mathrm{H}), 1.99(\mathrm{~m}, 2 \mathrm{H}), 0.90(\mathrm{~s}, 9 \mathrm{H}), 0.07(\mathrm{~s}, 6 \mathrm{H}) ;$

${ }^{13} \mathrm{C}$ NMR $\left(100 \mathrm{MHz}, \mathrm{CDCl}_{3}\right) \delta 62.3,36.2,25.9,18.3,3.6,-5.3$.

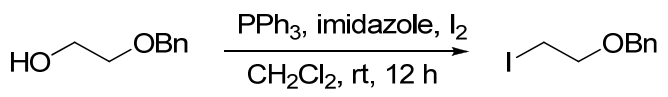

Compound 10²: The solution of 1-Benzyloxy-2-hydroxyethane (5.2 g, $34 \mathrm{mmol})$ in $\mathrm{CH}_{2} \mathrm{Cl}_{2}(100$ $\mathrm{mL}$ ) was treated consecutively with $\mathrm{PPh}_{3}(14.5 \mathrm{~g}, 51 \mathrm{mmol})$, imidazole $(3.5 \mathrm{~g}, 51 \mathrm{mmol})$, and iodine (12.9 g, $51 \mathrm{mmol})$. The reaction mixture was quenched with saturated aqueous sodium thiosulfate $(100 \mathrm{~mL})$ after $12 \mathrm{~h}$. The organic layers were separated, and the aqueous layer was extracted with $\mathrm{CH}_{2} \mathrm{Cl}_{2}(100 \mathrm{~mL} \times 2)$. The combined organic extracts were washed with brine, dried over anhydrous $\mathrm{Na}_{2} \mathrm{SO}_{4}$, and concentrated. The residue was purified by FCC $(\mathrm{PE}: \mathrm{EtOAc}=$

\footnotetext{
${ }^{1}$ Poole, L. B.; Zeng, B. B.; Knaggs, S. A.; Yakubu, M.; King, S. B. Bioconjuate. Chem. 2005, 16, 1624-1628.

2 Borbas, K. E.; Chandrashaker, V.; Muthiah,C.; Kee, H. L.; Holten, D.; Lindsey J. S. J. Org. Chem. 2008, 73 , 3145-3158.
} 
20:1) to yield 10 as colorless oil (7.2 g, 81\%). ${ }^{1} \mathrm{H}$ NMR (400 MHz, $\left.\mathrm{CDCl}_{3}\right) \delta \mathrm{H}$ 7.37-7.26 (m, 5H), $4.56(\mathrm{~s}, 2 \mathrm{H}), 3.72(\mathrm{t}, J=6.4 \mathrm{~Hz}, 2 \mathrm{H}), 3.26(\mathrm{t}, J=6.8 \mathrm{~Hz}, 2 \mathrm{H}) ;{ }^{13} \mathrm{C} \mathrm{NMR}\left(100 \mathrm{MHz}, \mathrm{CDCl}_{3}\right) \delta$ $137.7,128.4,127.8,127.7,72.8,70.7,2.9$.

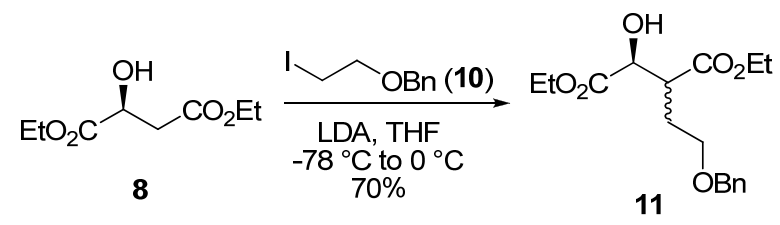

Compound 11: To the solution of $i$ - $\mathrm{Pr}_{2} \mathrm{NH}(6.7 \mathrm{~mL}, 48 \mathrm{mmol})$ in THF $(40 \mathrm{~mL})$ was added $n$-BuLi (2.0 M in THF, $22 \mathrm{~mL}, 44 \mathrm{mmol}$ ) at $-78^{\circ} \mathrm{C}$. The mixture was stirred at $-40{ }^{\circ} \mathrm{C}$ for $40 \mathrm{~min}$ before a solution of dimethyl L-malate $(3.8 \mathrm{~g}, 20 \mathrm{mmol})$ in THF $(5.0 \mathrm{~mL})$ was added over $3 \mathrm{~min}$ to the freshly prepared solution of LDA at $-78{ }^{\circ} \mathrm{C}$. After stirring at $-20{ }^{\circ} \mathrm{C}$ for $1 \mathrm{~h}$, HMPA $(18 \mathrm{~mL})$ was added at $-78^{\circ} \mathrm{C}$ and compound $\mathbf{1 0}(6.3 \mathrm{~g}, 24 \mathrm{mmol})$ in THF $(8 \mathrm{~mL})$ was introduced to the mixture over $3 \mathrm{~min}$. After stirring at $-20{ }^{\circ} \mathrm{C}$ for $12 \mathrm{~h}$, the solution was quenched with saturated aqueous $\mathrm{NH}_{4} \mathrm{Cl}(5 \mathrm{~mL})$ and diluted with EtOAc $(150 \mathrm{~mL})$. The organic phase was washed with water $(100$ $\mathrm{mL})$ and separated. The aqueous layer was extracted with EtOAc $(100 \mathrm{~mL} \times 3)$. The combined organic extracts were washed with brine, dried over anhydrous $\mathrm{Na}_{2} \mathrm{SO}_{4}$, and concentrated. Purification of the residue by FCC (PE : EtOAc $=4: 1)$ provided 11 as yellow oil $(4.55 \mathrm{~g}, 70 \%)$.<smiles>CCOC(=O)C(O)C(CCOc1ccccc1)C(=O)OCC</smiles>

11

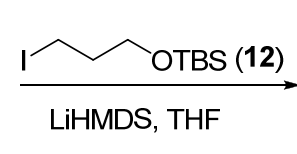

LiHMDS, THF

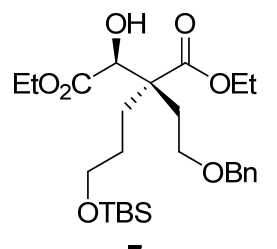

7

Compound 7: To the solution of HMDS (8.9 mL, $42 \mathrm{mmol})$ in THF (10 mL) was added $n$-BuLi (2.0 $\mathrm{M}$ in THF, $20 \mathrm{~mL}, 40 \mathrm{mmol}$ ) at $-78^{\circ} \mathrm{C}$, and the mixture was stirred at $-20^{\circ} \mathrm{C}$ for $40 \mathrm{~min}$ before a solution of compound $11(3.24 \mathrm{~g}, 10 \mathrm{mmol})$ in THF $(5.0 \mathrm{~mL})$ was added to the freshly prepared solution of LiHMDS at $-78^{\circ} \mathrm{C}$. After the mixture was stirred at $-5^{\circ} \mathrm{C}$ for $1 \mathrm{~h}$, compound $12(9 \mathrm{~g}, 30 \mathrm{mmol})$ in $10 \mathrm{~mL}$ THF was added over $3 \mathrm{~min}$ at $-78{ }^{\circ} \mathrm{C}$. Stirring continued at $-78^{\circ} \mathrm{C}$ for $2 \mathrm{~h}$ before the mixture was warmed to $-5{ }^{\circ} \mathrm{C}$ over $12 \mathrm{~h}$. Then the solution was quenched with saturated aqueous $\mathrm{NH}_{4} \mathrm{Cl}(5 \mathrm{~mL})$, diluted with EtOAc $(150 \mathrm{~mL})$ and water $(100 \mathrm{~mL})$. The organic 
phase was separated and the aqueous layer was extracted with EtOAc $(120 \mathrm{~mL} \times 3)$. The combined organic extracts were washed with brine, dried over anhydrous $\mathrm{Na}_{2} \mathrm{SO}_{4}$, and concentrated. Purification of the residue by FCC $(\mathrm{PE}:$ EtOAc $=10: 1-4: 1)$ provided 7 as yellow oil $(1.98 \mathrm{~g}, 40 \%)$ with $810 \mathrm{mg}$ compound 11 recovered. $[\alpha]_{\mathrm{D}}^{20}+7.4\left(c \quad 0.3, \mathrm{CHCl}_{3}\right) ;{ }^{1} \mathrm{H}$ NMR $(400$ $\left.\mathrm{MHz}, \mathrm{CDCl}_{3}\right) \delta 7.34-7.26(\mathrm{~m}, 5 \mathrm{H}), 4.48(\mathrm{~s}, 2 \mathrm{H}), 4.46(\mathrm{~d}, J=7.2 \mathrm{~Hz}, 1 \mathrm{H}), 4.20(\mathrm{q}, J=7.2 \mathrm{~Hz}, 2 \mathrm{H})$, $4.12(\mathrm{q}, J=7.2 \mathrm{~Hz}, 2 \mathrm{H}), 3.86(\mathrm{~d}, J=7.2 \mathrm{~Hz}, 1 \mathrm{H}), 3.67-3.59(\mathrm{~m}, 2 \mathrm{H}), 3.56$ (t, $J=6.4 \mathrm{~Hz}, 2 \mathrm{H})$, 2.14-2.02 (m, 2H), 1.81-1.64 (m, 2H), 1.57-1.46 (m, 2H), $1.27(\mathrm{t}, J=7.2 \mathrm{~Hz}, 3 \mathrm{H}), 1.23(\mathrm{t}, J=7.2$ $\mathrm{Hz}, 3 \mathrm{H}), 0.88(\mathrm{~s}, 9 \mathrm{H}), 0.03(\mathrm{~s}, 6 \mathrm{H}) ;{ }^{13} \mathrm{C} \mathrm{NMR}\left(100 \mathrm{MHz}, \mathrm{CDCl}_{3}\right) \delta 174.1,172.7,138.0,128.3$, 127.7, 127.6, 74.5, 73.1, 66.6, 63.3, 61.6, 60.9, 51.8, 32.3, 29.0, 27.5, 25.9, 18.3, 14.1, -5.3; IR (KBr) 3508.7, 2954.5, 2932.0, 2858.4, 1733.1, 1465.0, 1367.2, 1253.1, 1202.9, 1097.8, 1028.5, $838.1 \mathrm{~cm}^{-1}$; HRMS (ESI) $\mathrm{m} / z$ calcd for $\mathrm{C}_{26} \mathrm{H}_{45} \mathrm{O}_{7} \mathrm{Si}(\mathrm{M}+\mathrm{H})^{+}$497.2929; found 497.2933.
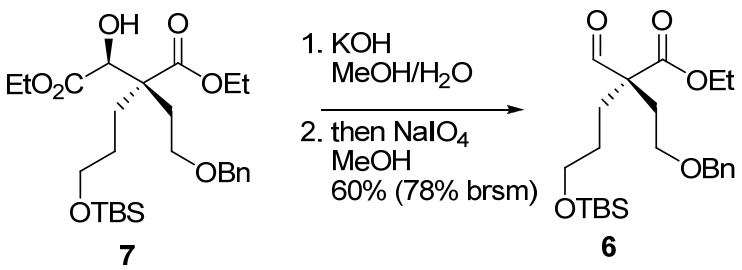

Compound 6: To the solution of compound 7 (500 mg, $1.0 \mathrm{mmol})$ in $\mathrm{MeOH}(4.5 \mathrm{~mL})$ was added successively $\mathrm{H}_{2} \mathrm{O}(0.3 \mathrm{~mL})$ and $\mathrm{KOH}(190 \mathrm{mg}, 3.4 \mathrm{mmol})$. After stirring for 3 days, the reaction mixture was diluted with EtOAc $(30 \mathrm{~mL})$ and $\mathrm{H}_{2} \mathrm{O}(30 \mathrm{~mL})$. Then $5 \%$ aqueous $\mathrm{HCl}$ was added to the solution drop by drop until the $\mathrm{pH}$ value of the aqueous phase was between 2 and 3 . The organic layer was separated, and the aqueous layer was extracted with EtOAc $(30 \mathrm{~mL} \times 3)$. The combined organic extracts were washed with brine, dried over anhydrous $\mathrm{Na}_{2} \mathrm{SO}_{4}$, and concentrated. To the solution of the residue in $\mathrm{MeOH}(10 \mathrm{~mL})$ was added $\mathrm{NaIO}_{4}(640 \mathrm{mg}, 3$ mmol), and the reaction mixture was shielded from light with aluminum foil for $18 \mathrm{~h}$ before the solution was dilued with EtOAc $(30 \mathrm{~mL})$ and water $(20 \mathrm{~mL})$. The organic layer was separated and the aqueous layer was extracted with EtOAc $(30 \mathrm{~mL} \times 3)$. The combined organic extracts were washed with brine, dried over anhydrous $\mathrm{Na}_{2} \mathrm{SO}_{4}$, and concentrated. Purification of the residue by FCC $(\mathrm{PE}: \mathrm{EtOAc}=10: 1)$ provided 6 as colorless oil $(253 \mathrm{mg}, 60 \%) \cdot[\alpha]_{\mathrm{D}}^{20}+6.8\left(c 0.3, \mathrm{CHCl}_{3}\right)$; ${ }^{1} \mathrm{H}$ NMR (400 MHz, $\left.\mathrm{CDCl}_{3}\right) \delta 9.85(\mathrm{~s}, 1 \mathrm{H}), 7.34-7.26(\mathrm{~m}, 5 \mathrm{H}), 4.41$ (s, 2H), 4.16 (q, $J=7.2 \mathrm{~Hz}$, 
2H), 3.59-3.43 (m, 4H), 2.23-2.10 (m, 2H), $1.93(\mathrm{~m}, 1 \mathrm{H}), 1.80(\mathrm{~m}, 1 \mathrm{H}), 1.48-1.35(\mathrm{~m}, 2 \mathrm{H}), 1.23(\mathrm{t}$, $J=7.2 \mathrm{~Hz}, 3 \mathrm{H}), 0.88(\mathrm{~s}, 9 \mathrm{H}), 0.03(\mathrm{~s}, 6 \mathrm{H}) ;{ }^{13} \mathrm{C} \mathrm{NMR}\left(100 \mathrm{MHz}, \mathrm{CDCl}_{3}\right) \delta 200.4,171.9,137.9$, $128.3,127.62,127.57,73.0,65.9,62.8,61.2,58.9,33.7,29.8,27.6,25.9,18.2,14.1,-5.4 ;$ IR (KBr), 2954.5, 2931.6, 2858.7, 1722.4, 1464.6, 1253.8, 1202.0, 1099.4, 837.6, $446.0 \mathrm{~cm}^{-1}$; HRMS (ESI) $m / z$ calcd for $\mathrm{C}_{23} \mathrm{H}_{42} \mathrm{NO}_{5} \mathrm{Si}\left(\mathrm{M}+\mathrm{NH}_{4}\right)^{+} 440.2827$; found 440.2829 .

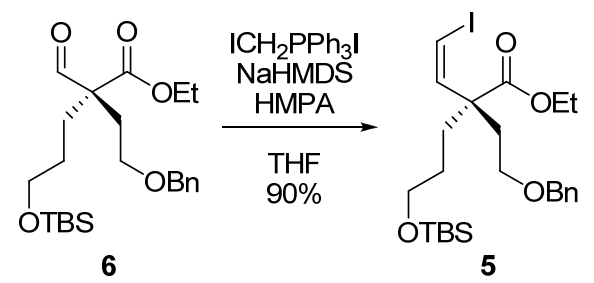

Compound 5: To the solution of Iodomethyltriphenylphosphonium iodide (106 mg, $2.00 \mathrm{mmol})$ ) in THF $(16 \mathrm{~mL})$ was added NaHMDS $(1.0 \mathrm{M}$ in THF, $1.96 \mathrm{~mL}, 1.96 \mathrm{mmol})$ at $0{ }^{\circ} \mathrm{C}$ dropwise over $1 \mathrm{~min}$. After stirring at $0{ }^{\circ} \mathrm{C}$ for $30 \mathrm{~min}$, HMPA $(8.6 \mathrm{~mL}, 4.8 \mathrm{mmol})$ was added in one portion and the reaction mixture was cooled to $-100{ }^{\circ} \mathrm{C}$. Compound $6(168 \mathrm{mg}, 0.40 \mathrm{mmol})$ in THF $(3 \mathrm{~mL})$ was added over $2 \mathrm{~min}$. The stirring was continued for $0.5 \mathrm{~h}$ before the reaction mixture was diluted with EtOAc $(30 \mathrm{~mL})$ and quenched with saturated $\mathrm{NH}_{4} \mathrm{Cl}(10 \mathrm{~mL})$. Then water $(20 \mathrm{~mL})$ was added and the organic layer was separated. The aqueous layer was extracted with EtOAc (30 $\mathrm{mL} \times 3$ ). The combined organic extracts were washed with brine, dried over anhydrous $\mathrm{Na}_{2} \mathrm{SO}_{4}$, and concentrated. Purification of the residue by FCC $(\mathrm{PE}:$ EtOAc $=25: 1)$ provided $\mathbf{5}$ as colorless oil (197 mg, 90\%). [ $\alpha]_{\mathrm{D}}^{20}-10.40\left(c 0.02, \mathrm{CHCl}_{3}\right) ;{ }^{1} \mathrm{H}$ NMR $\left(400 \mathrm{MHz}, \mathrm{CDCl}_{3}\right) \delta$ 7.32-7.26 (m, 5H), $6.94(\mathrm{~d}, J=8.8,1 \mathrm{H}), 6.39(\mathrm{~d}, J=8.4 \mathrm{~Hz}, 1 \mathrm{H}) 4.43(\mathrm{~s}, 2 \mathrm{H}), 4.14-4.09(\mathrm{~m}, 2 \mathrm{H}), 3.56(\mathrm{t}, J=6.4 \mathrm{~Hz}$, 2H), 3.47-3.41 (m, 2H), $2.46(\mathrm{~m}, 1 \mathrm{H}), 2.25(\mathrm{~m}, 1 \mathrm{H}), 2.17(\mathrm{~m}, 1 \mathrm{H}), 1.87(\mathrm{~m}, 1 \mathrm{H}), 1.43-1.26(\mathrm{~m}$, 2H), $\left.1.22(\mathrm{t}, J=6.8 \mathrm{~Hz}, 3 \mathrm{H}), 0.89(\mathrm{~s}, 9 \mathrm{H}), 0.04(\mathrm{~s}, 6 \mathrm{H}) ;{ }^{13} \mathrm{C} \mathrm{NMR} \mathrm{(100} \mathrm{MHz,} \mathrm{CDCl}_{3}\right) \delta 174.5$, $141.2,138.3,128.2,127.6,127.5,79.2,72.9,66.6,63.0,61.1,51.4,35.0,32.7,27.9,25.9,18.3$, 14.1, -5.3; IR (KBr) 433.5, 2954.8, 2930.77, 2857.7, 1729.9, 1455.6, 1254.7, 1195.9, 1100.0, 827.2, 776.8 $\mathrm{cm}^{-1}$; HRMS (ESI) $\mathrm{m} / z$ calcd for $\mathrm{C}_{24} \mathrm{H}_{40} \mathrm{IO}_{4} \mathrm{Si}(\mathrm{M}+\mathrm{H})^{+} 547.1735$; found 547.1730. 


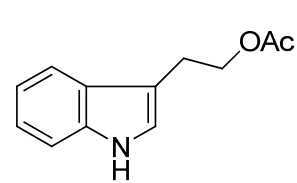

9

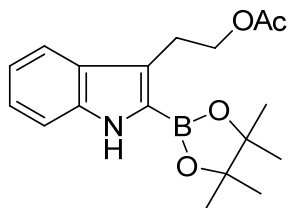

4

Compound 4: To a solution of 3-(2-acetoxyethyl)indole $(203 \mathrm{mg}, 1.00 \mathrm{mmol})$ in the mixture of hexane/DCM $(4 \mathrm{~mL} / 2 \mathrm{~mL})$ was added the pinacol borane $(217 \mathrm{uL}, 1.5 \mathrm{mmol})$ and 4,4'-di-tert-butyl-2,2'-dipyridyl $(2.7 \mathrm{mg}, 0.01 \mathrm{mmol})$. Later the solution was saturated with atmosphere of argon for $10 \mathrm{~min}$ before the catalyst (1, 5-cyclooctadiene)(methoxy)iridium(I) dimer $(3.3 \mathrm{mg}, 0.005 \mathrm{mmol}$ ) was added to the solution. The entire mixture was heated in an oil bath set to $50{ }^{\circ} \mathrm{C}$ and gradually turned into a brown solution. The mixture was allowed to cool to $23{ }^{\circ} \mathrm{C}$ after heating for $1 \mathrm{~h}$ and the volatiles were removed under reduced pressure. The crude mixture was purified by FCC (PE : EtOAc $=10: 1)$ to provide 4 as white solid $(197 \mathrm{mg}, 60 \%)$ with $51 \mathrm{mg}$ starting material recovered. ${ }^{1} \mathrm{H}$ NMR $\left(400 \mathrm{MHz}, \mathrm{CDCl}_{3}\right) \delta 8.44(\mathrm{~s}, 1 \mathrm{H}), 7.69(\mathrm{~d}, J=8.0 \mathrm{~Hz}$, 1H), $7.33(\mathrm{~d}, J=8.4 \mathrm{~Hz}, 1 \mathrm{H}), 7.22(\mathrm{t}, J=7.2 \mathrm{~Hz}, 1 \mathrm{H}), 7.09(\mathrm{t}, J=7.2 \mathrm{~Hz}, 1 \mathrm{H}), 4.32(\mathrm{t}, J=7.2 \mathrm{~Hz}$, $2 \mathrm{H}), 3.34(\mathrm{t}, J=7.2 \mathrm{~Hz}, 2 \mathrm{H}), 2.0(\mathrm{~s}, 3 \mathrm{H}), 1.35(\mathrm{~s}, 12 \mathrm{H}) ;{ }^{13} \mathrm{C} \mathrm{NMR}\left(100 \mathrm{MHz}, \mathrm{CDCl}_{3}\right) \delta 171.1$, 138.0, 128.4, 124.4, 123.7, 119.8, 119.3, 111.3, 83.9, 65.6, 24.8, 21.1; IR (KBr) 3400.3, 3057.9, 2977.3, 2926.0, 1737.6, 1554.2, 1386.9, 1319.2, 1265.7, 1250.9, 1142.2, 1026.6, 965.3, 858.5, 744.7, 709.8, $536.5 \mathrm{~cm}^{-1}$; HRMS (ESI) $\mathrm{m} / z$ calcd for $\mathrm{C}_{18} \mathrm{H}_{25} \mathrm{BNO}_{4}(\mathrm{M}+\mathrm{H})^{+}$330.1874; found 330.1880.

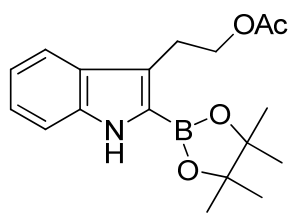

4

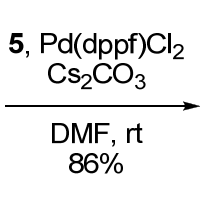

15

Compound 15: A solution of vinyl iodide $5(214 \mathrm{mg}, 0.39 \mathrm{mmol})$ in DMF $(10 \mathrm{~mL})$ was added successively compound $4(155 \mathrm{mg}, 0.47 \mathrm{mmol})$ and $\mathrm{Cs}_{2} \mathrm{CO}_{3}(390 \mathrm{mg}, 1.2 \mathrm{mmol})$. The solution was saturated with an atmosphere of argon for $15 \mathrm{~min}$ before $\mathrm{Pd}(\mathrm{dppf}) \mathrm{Cl}_{2}(28 \mathrm{mg}, 0.039 \mathrm{mmol})$ was added. The reaction mixture was stirred for $3 \mathrm{~h}$ before being diluted with EtOAc $(30 \mathrm{~mL})$ and 
neutralized with saturated aqueous $\mathrm{NH}_{4} \mathrm{Cl}(30 \mathrm{~mL})$. The organic phase was separated and the aqueous phase was extracted with EtOAc $(2 \times 30 \mathrm{~mL})$. The combined organic extracts were washed with brine, dried over anhydrous $\mathrm{Na}_{2} \mathrm{SO}_{4}$, and concentrated. Purification of the residue by FCC $(\mathrm{PE}: \mathrm{EtOAc}=10: 1)$ provided 15 as yellow oil $(193 \mathrm{mg}, 86 \%) .[\alpha]_{\mathrm{D}}^{20}-5.16,\left(c \mathrm{0.5}, \mathrm{CHCl}_{3}\right)$; ${ }^{1} \mathrm{H}$ NMR (400 MHz, $\left.\mathrm{CDCl}_{3}\right) \delta 8.49(\mathrm{~d}, J=7.6 \mathrm{~Hz}, 1 \mathrm{H}), 7.52(\mathrm{~d}, J=7.2 \mathrm{~Hz}, 1 \mathrm{H}), 7.32(\mathrm{~m}, 2 \mathrm{H})$, $7.07(\mathrm{~d}, J=7.2 \mathrm{~Hz}, 1 \mathrm{H}), 7.00(\mathrm{t}, J=7.6 \mathrm{~Hz}, 2 \mathrm{H}), 6.94(\mathrm{~d}, J=7.2 \mathrm{~Hz}, 2 \mathrm{H}), 6.79(\mathrm{~d}, J=10.0 \mathrm{~Hz}$, 1H), 5.79 (d, $J=10.0 \mathrm{~Hz}, 1 \mathrm{H}), 4.26-4.15(\mathrm{~m}, 4 \mathrm{H}), 3.56-3.39(\mathrm{~m}, 4 \mathrm{H}), 3.06$ (t, $J=7.2 \mathrm{~Hz}, 2 \mathrm{H})$, $2.69(\mathrm{~m}, 1 \mathrm{H}), 2.13(\mathrm{~m}, 1 \mathrm{H}), 2.01(\mathrm{~s}, 3 \mathrm{H}), 1.82(\mathrm{~m}, 1 \mathrm{H}), 1.68(\mathrm{~m}, 1 \mathrm{H}), 1.42(\mathrm{~m}, 1 \mathrm{H}), 1.27(\mathrm{~m}, 1 \mathrm{H})$, $0.85(\mathrm{~s}, 9 \mathrm{H}),-0.017(\mathrm{~s}, 3 \mathrm{H}),-0.026(\mathrm{~s}, 3 \mathrm{H}) ;{ }^{13} \mathrm{C} \mathrm{NMR}\left(100 \mathrm{MHz}, \mathrm{CDCl}_{3}\right) \delta 172.8,170.9,137.8$, 134.4, 133.0, 132.6, 130.6, 127.9, 127.2, 127.1, 125.5, 124.0, 118.3, 116.7, 115.8, 113.5, 73.0, $67.1,63.6,62.8,49.5,40.0,37.6,27.8,25.9,23.3,20.9,18.3,-5.35,-5.37$; IR (KBr) 3736.8, 2953.5, 2917.0, 2851.9, 1738.9, 1458.5, 1376.4, 1236.9, 1101.0, 835.9, 491.1 $\mathrm{cm}^{-1}$; HRMS (ESI) $m / z$ calcd for $\mathrm{C}_{34} \mathrm{H}_{46} \mathrm{NO}_{5} \mathrm{Si}(\mathrm{M}+\mathrm{H})^{+} 576.3140$; found 576.3129 .
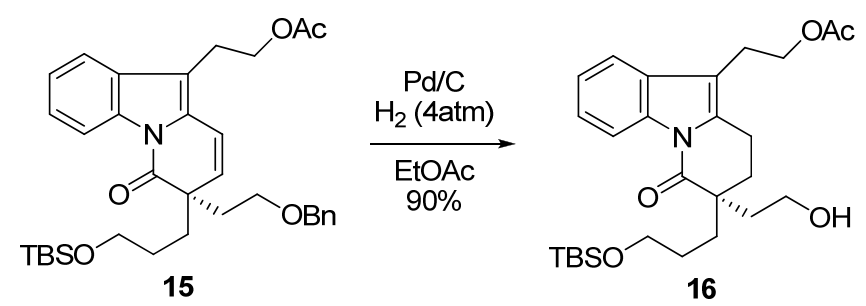

Compound 16: Compound 15 (57 mg, $0.1 \mathrm{mmol})$ was dissolved in EtOAc $(10 \mathrm{~mL})$ and transfered to a hydrogen tank. Palladium on carbon $(10 \% \mathrm{Pd}, 0.019 \mathrm{mmol} \mathrm{Pd}, 20 \mathrm{mg})$ was added to the tank and the suspension was stirred under an atmosphere of hydrogen (4 atm) for $2 \mathrm{~h}$. Then catalyst was removed by filtering through a Celite pad, washing the pad with EtOAc. The solvent was removed by rotary evaporation. Purification of the residue by FCC $(\mathrm{PE}:$ EtOAc $=1: 2)$ provided 16 as colorless oil (44 mg, 90\%). $[\alpha]_{\mathrm{D}}^{20}-4.80,\left(c \mathrm{c} .5, \mathrm{CHCl}_{3}\right) ;{ }^{1} \mathrm{H} \mathrm{NMR}\left(400 \mathrm{MHz}, \mathrm{CDCl}_{3}\right) \delta 8.45$ (m, 1H), $7.48(\mathrm{~m}, 1 \mathrm{H}), 7.31-7.27(\mathrm{~m}, 2 \mathrm{H}), 4.26(\mathrm{t}, J=7.2 \mathrm{~Hz}, 2 \mathrm{H}), 3.85(\mathrm{~m}, 1 \mathrm{H}), 3.80(\mathrm{~m}, 1 \mathrm{H})$, $3.61(\mathrm{t}, J=6.4 \mathrm{~Hz}, 2 \mathrm{H}), 3.06-2.95(\mathrm{~m}, 4 \mathrm{H}), 2.17-2.09(\mathrm{~m}, 2 \mathrm{H}), 2.04(\mathrm{~m}, 1 \mathrm{H}), 2.03(\mathrm{~s}, 3 \mathrm{H}), 1.96(\mathrm{~m}$, 1H), 1.87-1.79 (m, 2H), 1.61-1.50 (m, 3H), 0.85 (s, 9H), $0.03(\mathrm{~s}, 3 \mathrm{H}), 0.01(\mathrm{~s}, 3 \mathrm{H}) ;{ }^{13} \mathrm{C}$ NMR $(100$ $\left.\mathrm{MHz}, \mathrm{CDCl}_{3}\right) \delta 174.8,170.9,135.0,134.1,130.4,124.3,123.9,117.8,116.8,112.5,63.4,62.9$, $58.9,49.6,45.2,37.8,31.6,29.8,27.1,25.9,23.5,23.3,21.0,18.3,17.9,-5.35,-5.37$; IR (KBr) 
2969.2, 2865.9, 1734.1, 1721.1, 1621.8, 1447.9, 1367.1, 1326.4, 1228.5, 1216.9, 1152.3, 1114.3, 1086.2, 744.8, $525.7 \mathrm{~cm}^{-1}$; HRMS (ESI) $m / z$ calcd for $\mathrm{C}_{27} \mathrm{H}_{42} \mathrm{NO}_{5} \mathrm{Si}(\mathrm{M}+\mathrm{H})^{+}$488.2827; found 488.2835 .
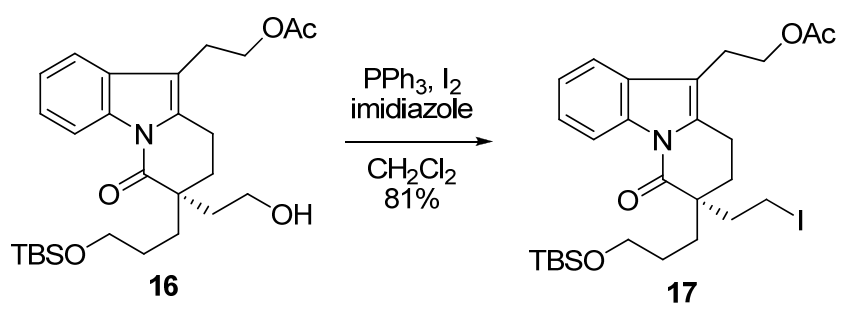

Compund 17: To a solution of compound 16 (48 mg, $0.1 \mathrm{mmol})$ in $\mathrm{CH}_{2} \mathrm{Cl}_{2}(5 \mathrm{~mL})$ was treated consecutively with $\mathrm{PPh}_{3}(52 \mathrm{mg}, 0.2 \mathrm{mmol}$ ), imidazole (14 mg, $0.2 \mathrm{mmol}$ ), and iodine (38 $\mathrm{mg}$, $0.15 \mathrm{mmol}$ ). After $20 \mathrm{~min}$, the reaction mixture was quenched with saturated aqueous sodium thiosulfate $(10 \mathrm{~mL})$. The organic layers were separated, and the aqueous layer was extracted with EtOAc $(15 \mathrm{~mL} \times 3)$. The combined organic phase was washed with brine, dried over anhydrous $\mathrm{Na}_{2} \mathrm{SO}_{4}$, and concentrated. The residue was purified by FCC $(\mathrm{PE}:$ EtOAc $=10: 1)$ to yield $\mathbf{1 7}$ as colorless oil (48.5 mg, 81\%). $[\alpha]_{\mathrm{D}}^{25}+23.2\left(c 0.1, \mathrm{CHCl}_{3}\right) ;{ }^{1} \mathrm{H} \mathrm{NMR}\left(400 \mathrm{MHz}, \mathrm{CDCl}_{3}\right) \delta 8.45(\mathrm{~m}$, 1H), $7.49(\mathrm{~m}, 1 \mathrm{H}), 7.31-7.27(\mathrm{~m}, 2 \mathrm{H}), 4.25(\mathrm{t}, J=7.2 \mathrm{~Hz}, 2 \mathrm{H}), 3.61(\mathrm{t}, J=6.4 \mathrm{~Hz}, 2 \mathrm{H}), 3.31-3.21$ $(\mathrm{m}, 2 \mathrm{H}), 3.03-2.95(\mathrm{~m}, 4 \mathrm{H}), 2.46(\mathrm{~m}, 1 \mathrm{H}), 2.32(\mathrm{~m}, 1 \mathrm{H}), 2.06-2.03(\mathrm{~m}, 2 \mathrm{H}), 2.04(\mathrm{~s}, 3 \mathrm{H})$ $1.82-1.78(\mathrm{~m}, 2 \mathrm{H}), 1.60-1.53(\mathrm{~m}, 2 \mathrm{H}), 0.85(\mathrm{~s}, 12 \mathrm{H}), 0.03(\mathrm{~s}, 3 \mathrm{H}), 0.01(\mathrm{~s}, 3 \mathrm{H}) ;{ }^{13} \mathrm{C}$ NMR $(100$ $\left.\mathrm{MHz}, \mathrm{CDCl}_{3}\right) \delta 172.3,170.9,134.9,133.7,130.3,124.4,123.9,117.8,116.7,112.6,63.4,62.8$, 48.3, 40.8, 31.1, 29.2, 27.1, 25.9, 23.5, 21.0, 18.2, 17.8, -1.1, -5.34, -5.36; IR (KBr) 3690.4, 2983.1, 2893.5, 1778.7, 1725.9, 1621.9, 1501.8, 1450.6, 1357.1, 1325.3, 1268.5, 1220.2, 1151.3, $1117.9,1088.9,1026.5,830.9,746.8,688.8,574.3,530.1 \mathrm{~cm}^{-1}$; HRMS (ESI) $\mathrm{m} / z$ calcd for $\mathrm{C}_{27} \mathrm{H}_{41} \mathrm{INO}_{4} \mathrm{Si}(\mathrm{M}+\mathrm{H})^{+}$598.1844; found 598.1832. 

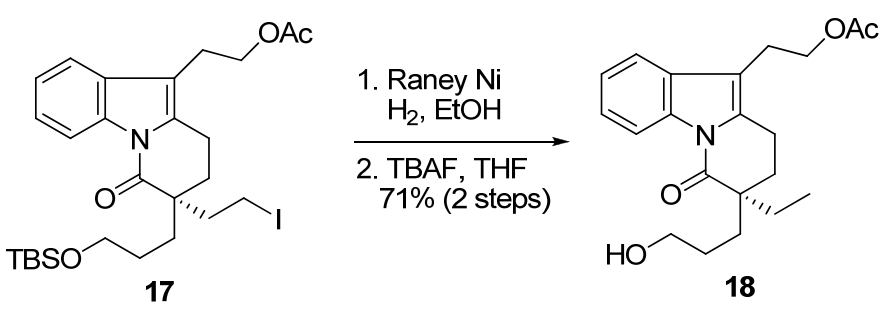

Compound 18: Compound 17 (30 mg, $0.05 \mathrm{mmol})$ was dissolved in EtOH $(5 \mathrm{~mL})$ and transfered to a hydrogen tank. An excess of the freshly made Raney nickel was added to the vigorously stirred solution. The suspension was stirred under an atmosphere of hydrogen (4 atm) for $4 \mathrm{~h}$ before the mixture was filtered through silica gel and washed with EtOAc. The solvent was evaporated to yield the reduced compound which was directly dissolved in THF $(3 \mathrm{~mL})$ and added with a solution of TBAF $(0.15 \mathrm{~mL}, 0.15 \mathrm{mmol})$. After stirring at room temperature for $6 \mathrm{~h}$, the volatiles were removed under reduced pressure and the residue was purified by FCC (PE : EtOAc $=1: 2)$ to provide 18 as colorless oil $(12.7 \mathrm{mg}, 71 \%)$. [ $\alpha]_{\mathrm{D}}^{25}-31.2\left(c 0.1, \mathrm{CHCl}_{3}\right) ;{ }^{1} \mathrm{H}$ NMR $(400$ $\left.\mathrm{MHz}, \mathrm{CDCl}_{3}\right) \delta 8.48(\mathrm{~m}, 1 \mathrm{H}), 7.49(\mathrm{~m}, 1 \mathrm{H}), 7.29-7.27(\mathrm{~m}, 2 \mathrm{H}), 4.26(\mathrm{t}, J=7.2 \mathrm{~Hz}, 2 \mathrm{H}), 3.65(\mathrm{t}, J$ $=6.0 \mathrm{~Hz}, 2 \mathrm{H}), 3.04-2.95(\mathrm{~m}, 4 \mathrm{H}), 2.05-2.02(\mathrm{~m}, 2 \mathrm{H}), 2.03(\mathrm{~s}, 3 \mathrm{H}), 1.97-1.67(\mathrm{~m}, 4 \mathrm{H}), 1.67-1.60$ (m, 2H), 1.48 (br s, 1H), 0.96 (t, $J=7.6 \mathrm{~Hz}, 3 \mathrm{H}) ;{ }^{13} \mathrm{C} \mathrm{NMR}\left(100 \mathrm{MHz}, \mathrm{CDCl}_{3}\right) \delta$ 173.9, 171.0, $134.9,134.3,130.3,124.2,123.7,117.7,116.7,112.1,63.5,63.0,46.1,31.1,28.6,28.4,27.3,23.5$, 21.0, 18.0. 8.3; IR (KBr) 2969.0, 2850.9, 1737.8, 1712.6, 1621.0, 1501.0, 1457.0, 1367.0, 1327.0, 1310.4, 1229.6, 1217.2, 1152.3, 1085.4, 1022.5, 752.9, 524.6, $512.6 \mathrm{~cm}^{-1}$; HRMS (ESI) $\mathrm{m} / z$ calcd for $\mathrm{C}_{21} \mathrm{H}_{28} \mathrm{NO}_{4}(\mathrm{M}+\mathrm{H})^{+}$358.2013; found 358.2020.
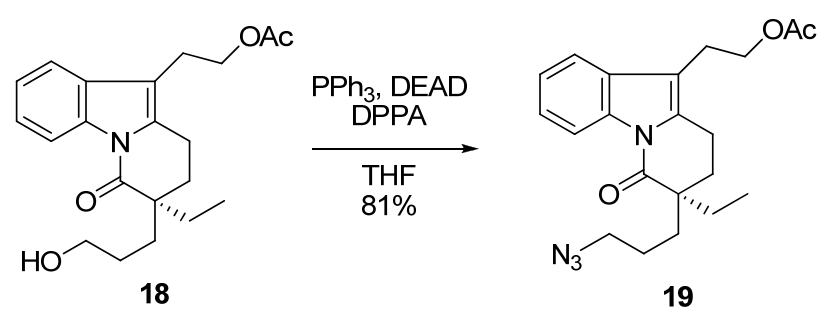

Compound 19: To a solution of alcohol 18 (28 mg, $0.078 \mathrm{mmol})$ in THF (5 mL) was added $\mathrm{PPh}_{3}$ (30 mg, $0.12 \mathrm{mmol})$, DPPA (25uL, $0.12 \mathrm{mmol})$, and DEAD (50 uL, $0.12 \mathrm{mmol}$ ) sequentially. The reaction mixture was stirred at room temperature for $3 \mathrm{~h}$ under Ar. The resulting solution was evaporated and the residue was purified by FCC $(\mathrm{PE}: \mathrm{EtOAc}=3: 1)$ to provide $\mathbf{1 9}$ as colorless oil

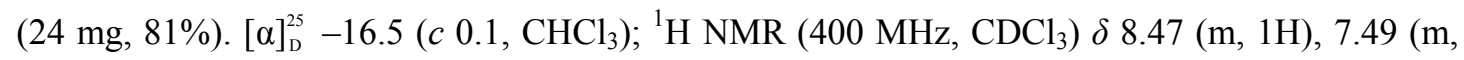


1H), 7.30-7.27 (m, 2H), $4.26(\mathrm{t}, J=7.2 \mathrm{~Hz}, 2 \mathrm{H}), 3.32(\mathrm{t}, J=6.4 \mathrm{~Hz}, 2 \mathrm{H}), 3.03-2.96(\mathrm{~m}, 4 \mathrm{H}), 2.03$ (s, 3H), 2.05-2.01 (m, 2H), 1.93-1.71 (m, 4H), 1.69-1.60 (m, 2H), $0.96(\mathrm{t}, J=7.6 \mathrm{~Hz}, 3 \mathrm{H}) ;{ }^{13} \mathrm{C}$ NMR $\left(100 \mathrm{MHz}, \mathrm{CDCl}_{3}\right) \delta 173.4,170.9,134.9,134.1,130.3,124.3,123.8,117.7,116.7,112.2$, $63.5,51.8,46.2,32.2,28.7,28.2,23.8,23.5,21.1,17.9,8.3$; IR (KBr) 3467.2, 2968.0, 2867.2, 2097.3, 1718.2, 1622.9, 1501.8, 1448.2, 1369.4, 1327.1, 1217.6, 1153.3, 1086.0, 744.7, 482.6, $462.8 \mathrm{~cm}^{-1}$; HRMS (ESI) $m / z$ calcd for $\mathrm{C}_{21} \mathrm{H}_{26} \mathrm{~N}_{4} \mathrm{O}_{3}(\mathrm{M}+\mathrm{H})^{+}$383.2078; found 383.2068.

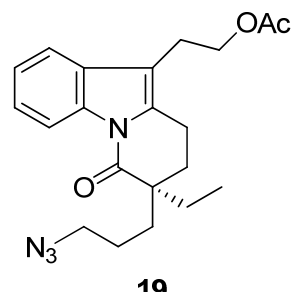

19

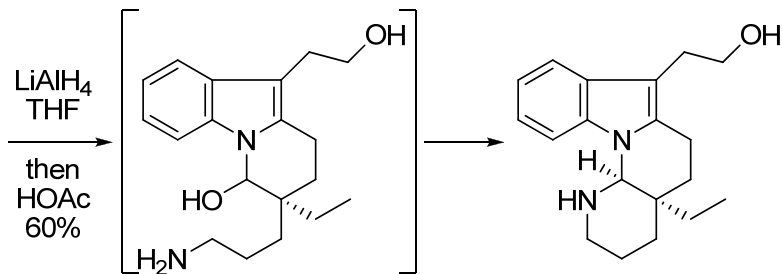

(-)-goniomitine (1)

(-)-Goniomitine: To a solution of azide $19(10 \mathrm{mg}, 0.026 \mathrm{mmol})$ in THF $(2 \mathrm{~mL})$ was added $\mathrm{LiAlH}_{4}(4 \mathrm{mg}, 0.1 \mathrm{mmol})$ at $0{ }^{\circ} \mathrm{C}$. The resulting solution was stirred at $0{ }^{\circ} \mathrm{C}$ for $30 \mathrm{~min}$ before being warmed to room temperature. After stirring at room temperature for $2 \mathrm{~h}$, the reaction was quenched with saturated aqueous potassium sodium. The mixture was extracted with EtOAc $(4 \times$ $20 \mathrm{~mL}$ ). The combined organic extracts were dried over $\mathrm{Na}_{2} \mathrm{SO}_{4}$, concentrated, and the crude product was treated with $2 \mathrm{~mL}$ of $\mathrm{AcOH}-\mathrm{THF}-\mathrm{H}_{2} \mathrm{O}$ solution $(3: 1: 1, \mathrm{~V} / \mathrm{V})$ at room temperature for $8 \mathrm{~h}$. The resulting mixture was treated with $20 \% \mathrm{NaOH}$ solution until $\mathrm{pH}>12$ and the solution was extracted with EtOAc $(5 \times 20 \mathrm{~mL})$. The combined organic extracts were dried over $\mathrm{Na}_{2} \mathrm{SO}_{4}$ and concentrated. The residue was purified by FCC $\left(\mathrm{DCM}: \mathrm{MeOH}=20: 1+1 \% \mathrm{Et}_{3} \mathrm{~N}\right)$ to provide goniomitine as pale yellow oil $(4.6 \mathrm{mg}, 60 \%)$. $[\alpha]_{\mathrm{D}}^{25}-80\left(c \mathrm{c} 0.46, \mathrm{CHCl}_{3}\right) ;{ }^{1} \mathrm{H} \mathrm{NMR}(400 \mathrm{MHz}$, $\left.\mathrm{CDCl}_{3}\right) \delta 7.52(\mathrm{~d}, J=7.6 \mathrm{~Hz}, 1 \mathrm{H}), 7.30(\mathrm{~d}, J=8.0 \mathrm{~Hz}, 1 \mathrm{H}), 7.14(\mathrm{t}, J=6.8 \mathrm{~Hz}, 1 \mathrm{H}), 7.08(\mathrm{t}, J=$ $7.2 \mathrm{~Hz}, 1 \mathrm{H}), 4.79(\mathrm{~s}, 1 \mathrm{H}), 3.83(\mathrm{t}, J=6.4 \mathrm{~Hz}, 2 \mathrm{H}), 3.06-3.00(\mathrm{~m}, 2 \mathrm{H}), 2.96-2.92(\mathrm{~m}, 2 \mathrm{H})$, 2.88-2.77 (m, 2H), $2.51(\mathrm{td}, \mathrm{J}=6.4,13.2 \mathrm{~Hz}, 1 \mathrm{H}), 1.91-1.87(\mathrm{~m}, 1 \mathrm{H}), 1.73-1.63(\mathrm{~m}, 1 \mathrm{H})$, 1.61-1.56 (m, 1H), 1.54-1.45 (m, 3H), 1.22-1.15 (m, 1H), $0.88(\mathrm{t}, \mathrm{J}=7.2 \mathrm{~Hz}, 3 \mathrm{H}) ;{ }^{13} \mathrm{C}$ NMR $(100$ $\left.\mathrm{MHz}, \mathrm{CDCl}_{3}\right) \delta 135.4,132.7,129.0,120.5,119.5,118.1,108.2,105.9,71.6,62.6,45.6,35.1,34.0$, 28.6, 27.7, 21.64, 21.58, 18.5, 7.1; IR (KBr) 2926.9, 2854.1, 1758.4, 1373.9, 1311.0, 1243.5, 1100.4, 1051.2, 835.3, 722.7, $605.9 \mathrm{~cm}^{-1}$; HRMS (ESI) $\mathrm{m} / z$ calcd for $\mathrm{C}_{19} \mathrm{H}_{27} \mathrm{~N}_{2} \mathrm{O}(\mathrm{M}+\mathrm{H})^{+}$ 299.2118; found 299.2119. The characterization data were consistent with those reported. 
${ }^{1} \mathrm{H}$ NMR of Compound 10 (400 MHz, $\mathrm{CDCl}_{3}$ )

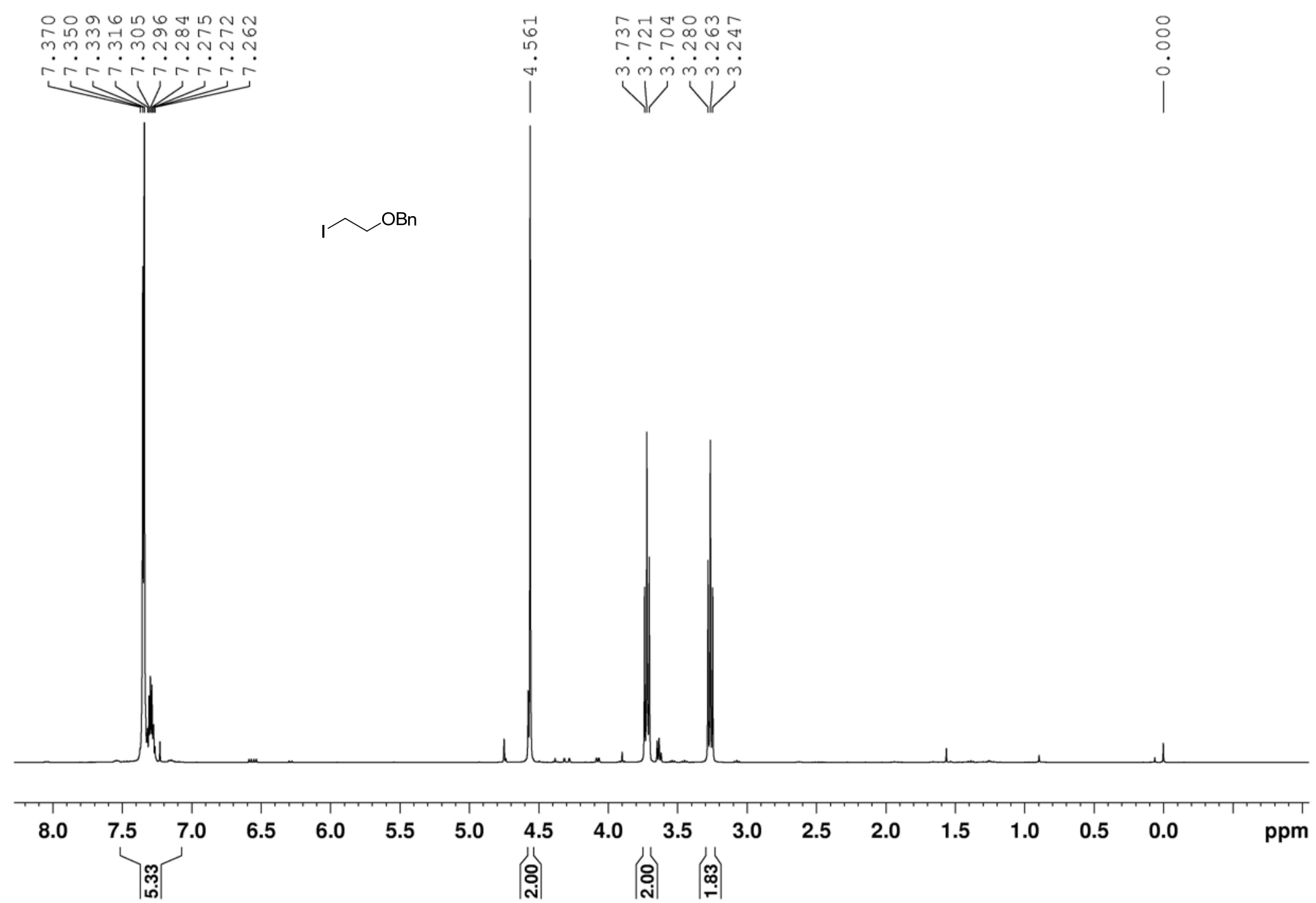


${ }^{13} \mathrm{C}$ NMR of Compound 10 (100 $\left.\mathrm{MHz}, \mathrm{CDCl}_{3}\right)$

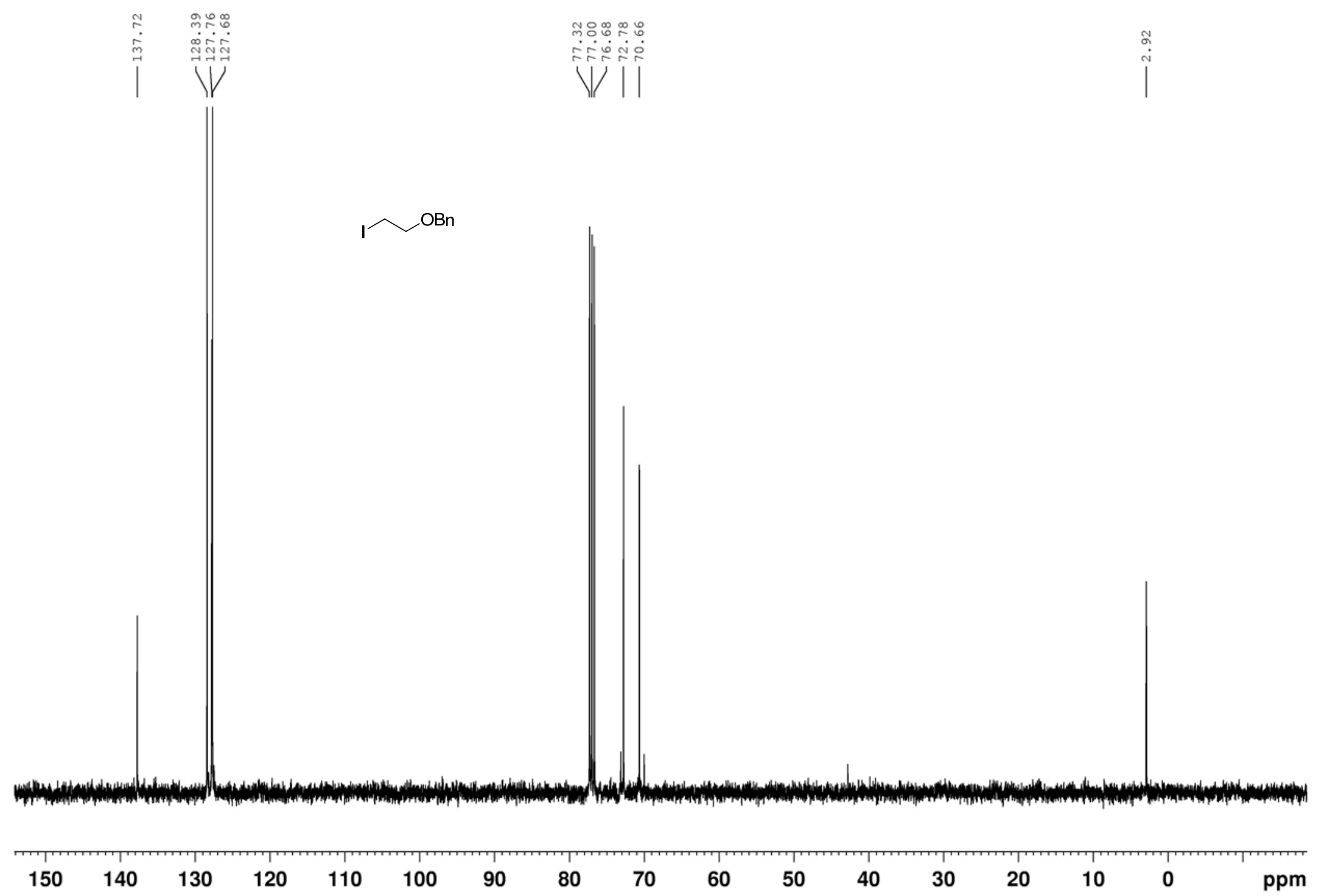


${ }^{1} \mathrm{H}$ NMR of Compound $12\left(400 \mathrm{MHz}, \mathrm{CDCl}_{3}\right)$

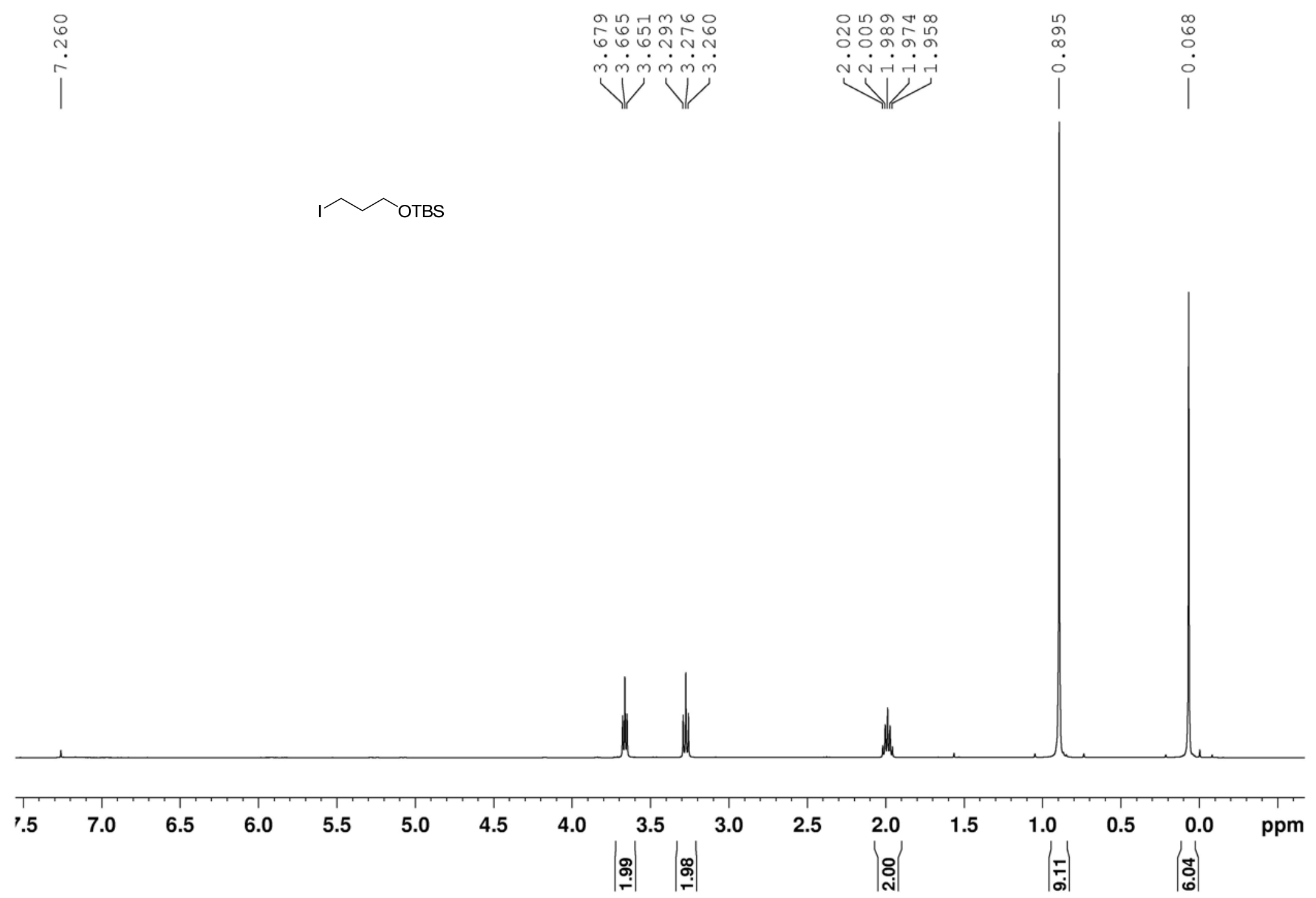


${ }^{13} \mathrm{C}$ NMR of Compound 12 (100 MHz, $\left.\mathrm{CDCl}_{3}\right)$

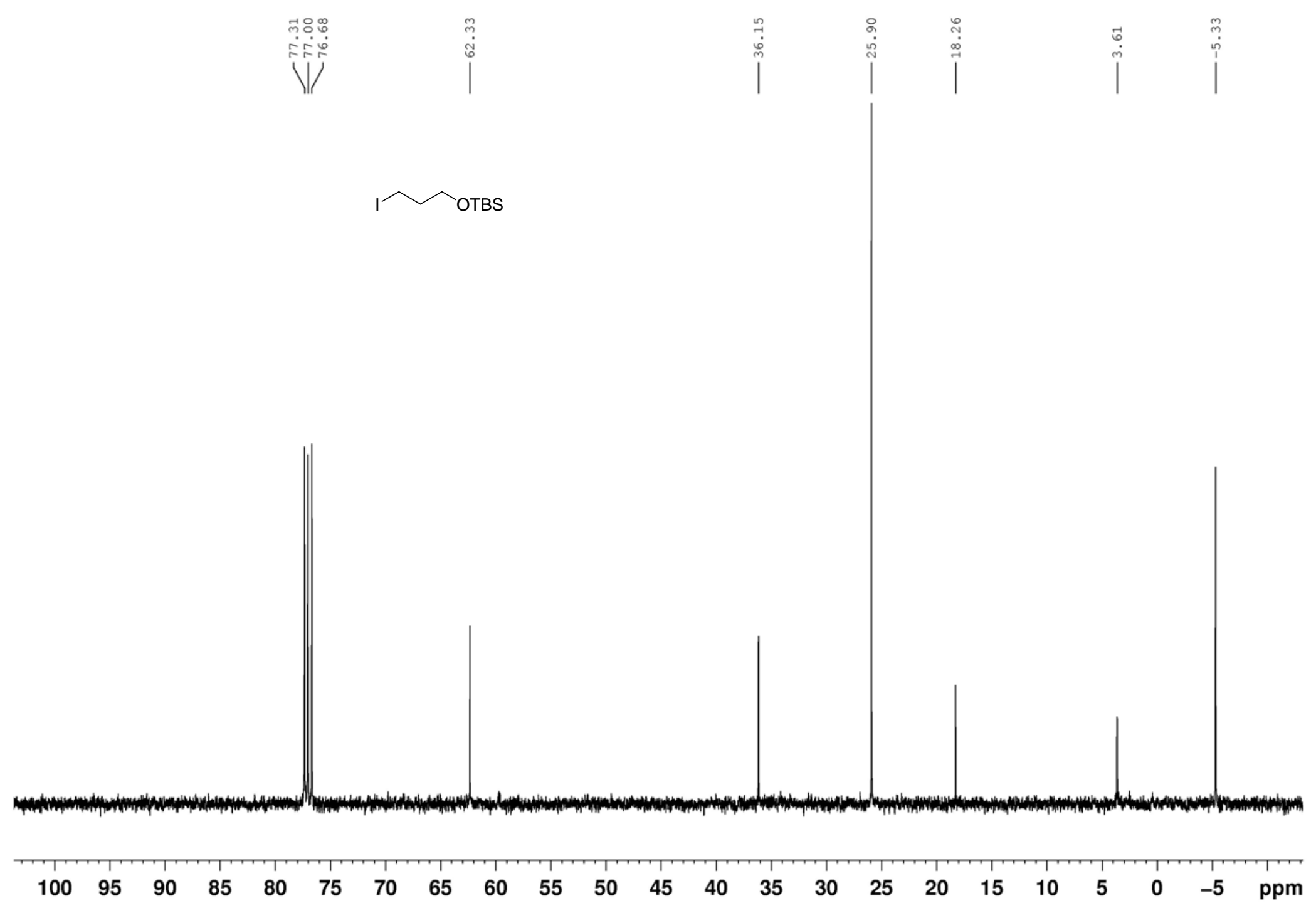


${ }^{1} \mathrm{H}$ NMR of Compound $7\left(400 \mathrm{MHz}, \mathrm{CDCl}_{3}\right)$

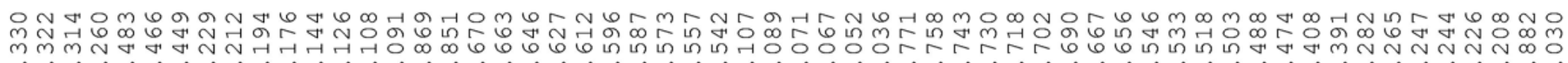
viriv<smiles>CCOC(=O)C(CCCO[Sb](C)(=O)c1ccccc1)(C(=O)OCC)[C@@H](O)CCOCc1ccccc1</smiles>

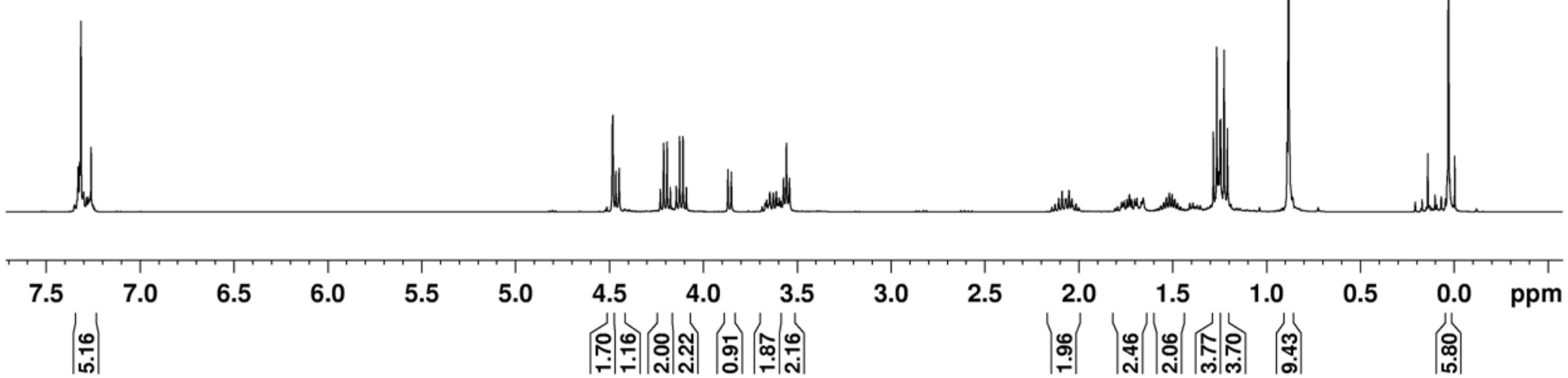


${ }^{13} \mathrm{C}$ NMR of Compound 7 (100 MHz, $\mathrm{CDCl}_{3}$ )
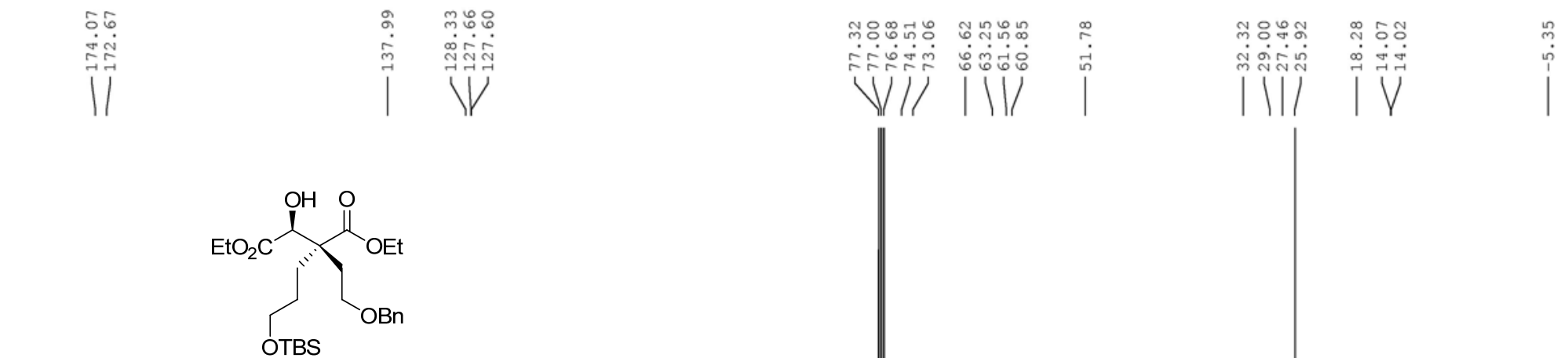

\section{.}

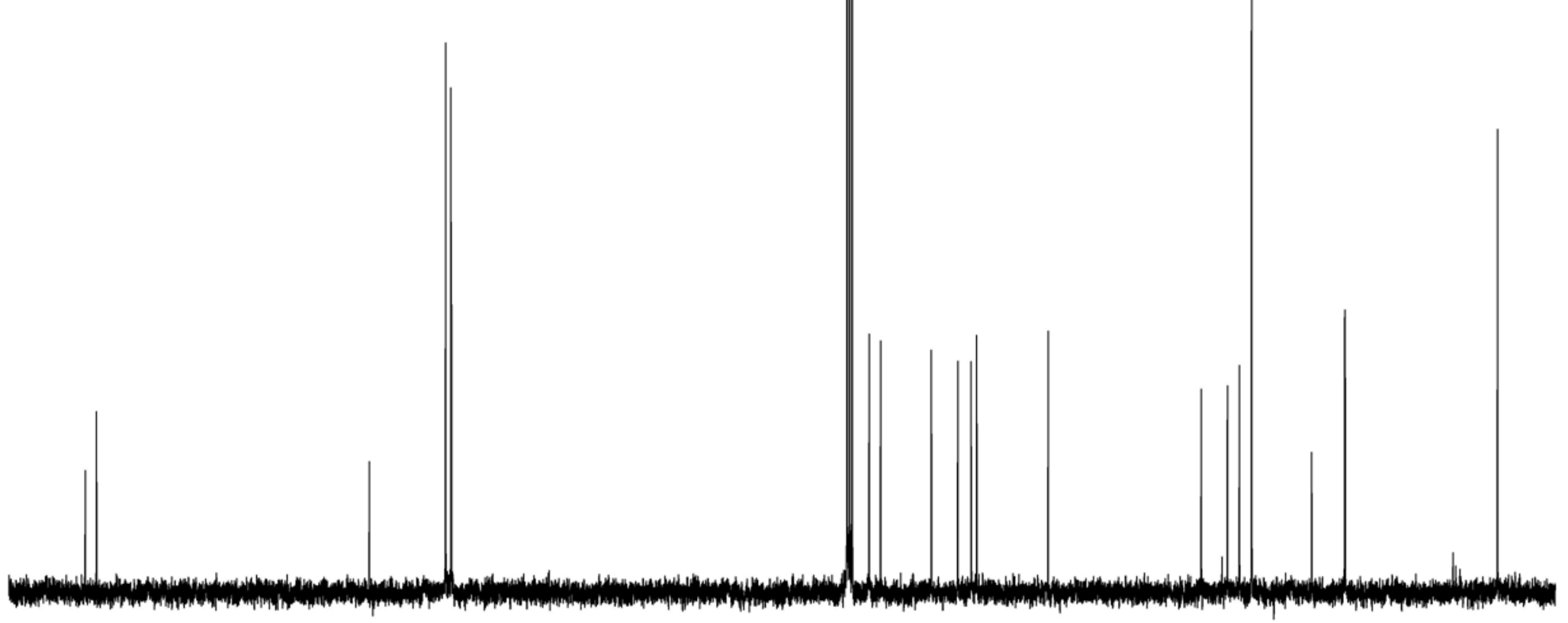


${ }^{1} \mathrm{H}$ NMR of Compound $6\left(400 \mathrm{MHz}, \mathrm{CDCl}_{3}\right)$

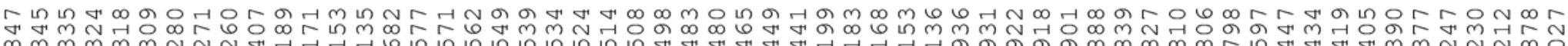

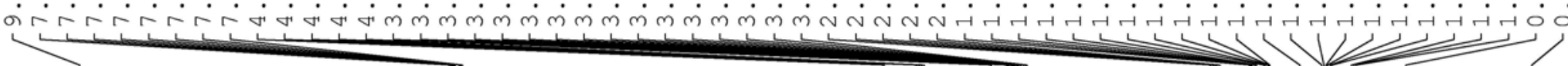
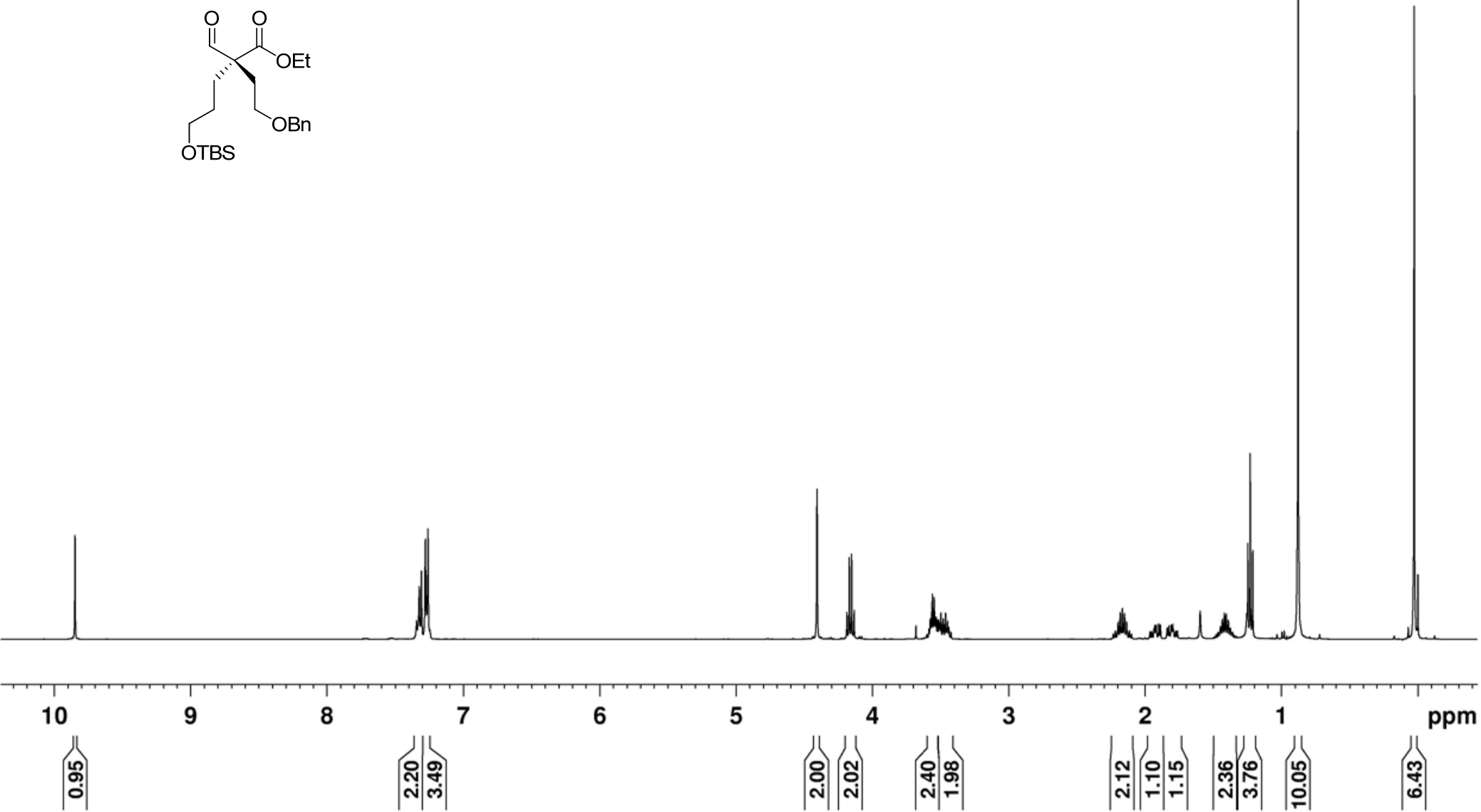
${ }^{13} \mathrm{C}$ NMR of Compound 6 (100 MHz, $\mathrm{CDCl}_{3}$ )
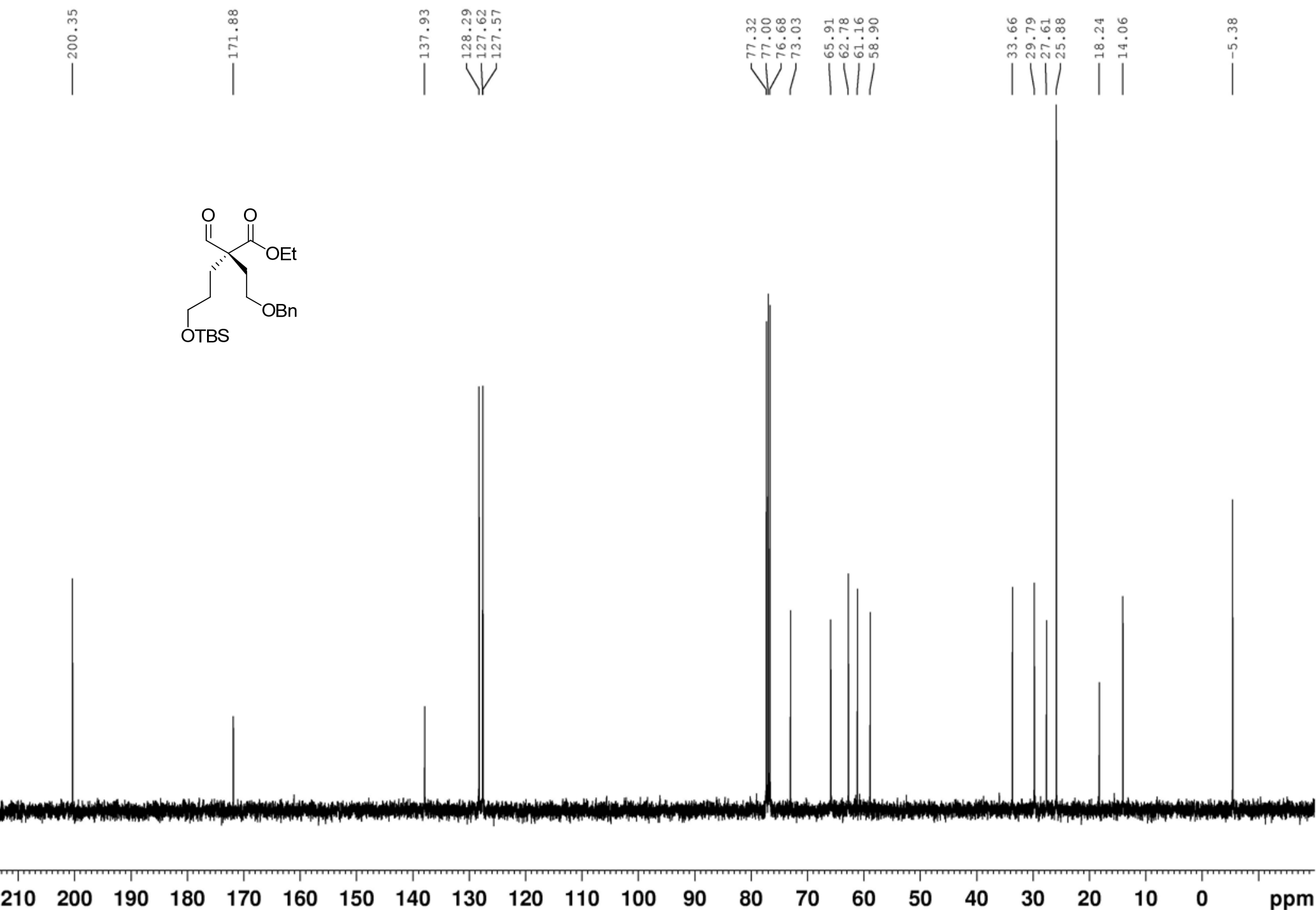
${ }^{1} \mathrm{H}$ NMR of Compound $5\left(400 \mathrm{MHz}, \mathrm{CDCl}_{3}\right)$

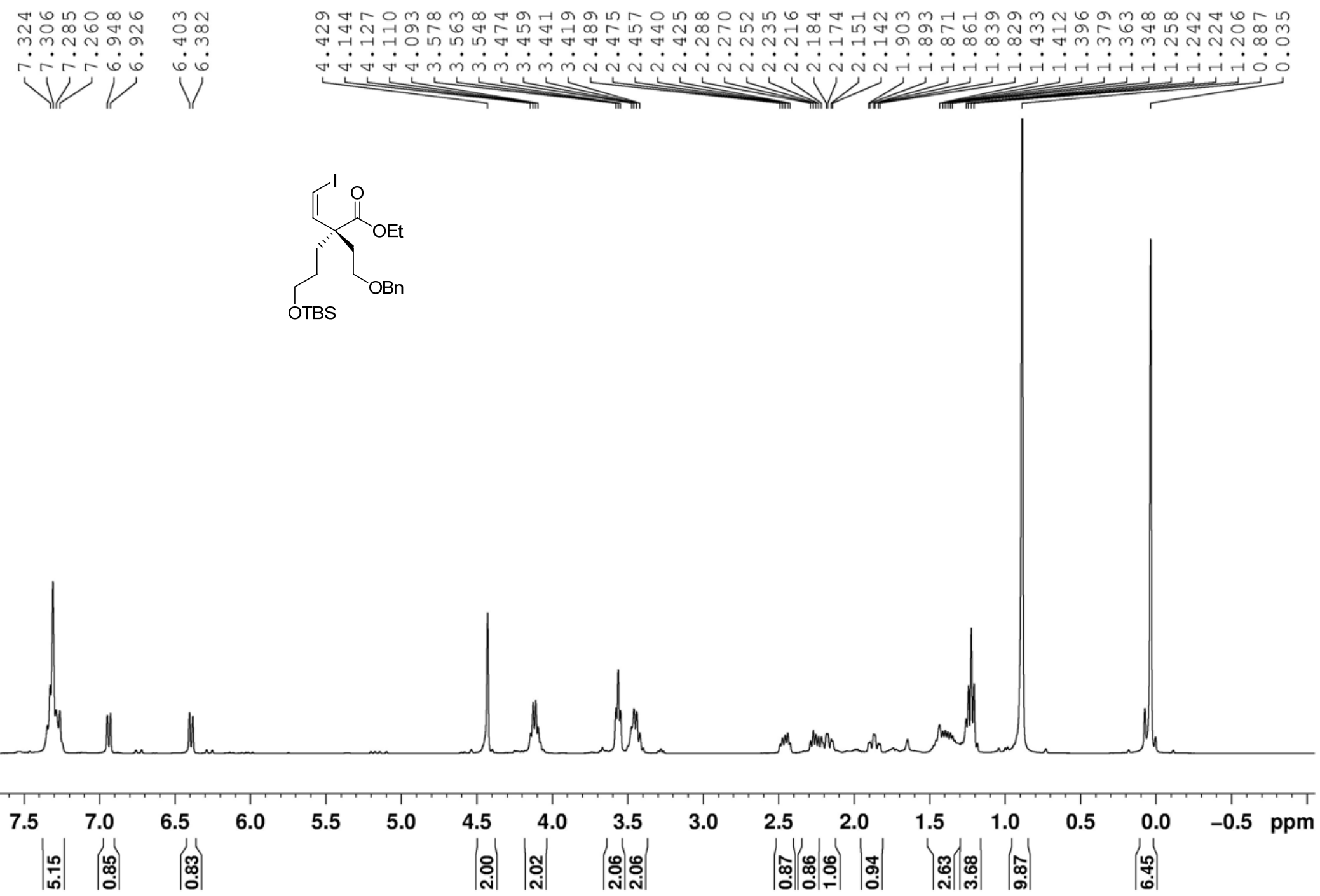


${ }^{13} \mathrm{C}$ NMR of Compound 5 (100 MHz, $\mathrm{CDCl}_{3}$ )

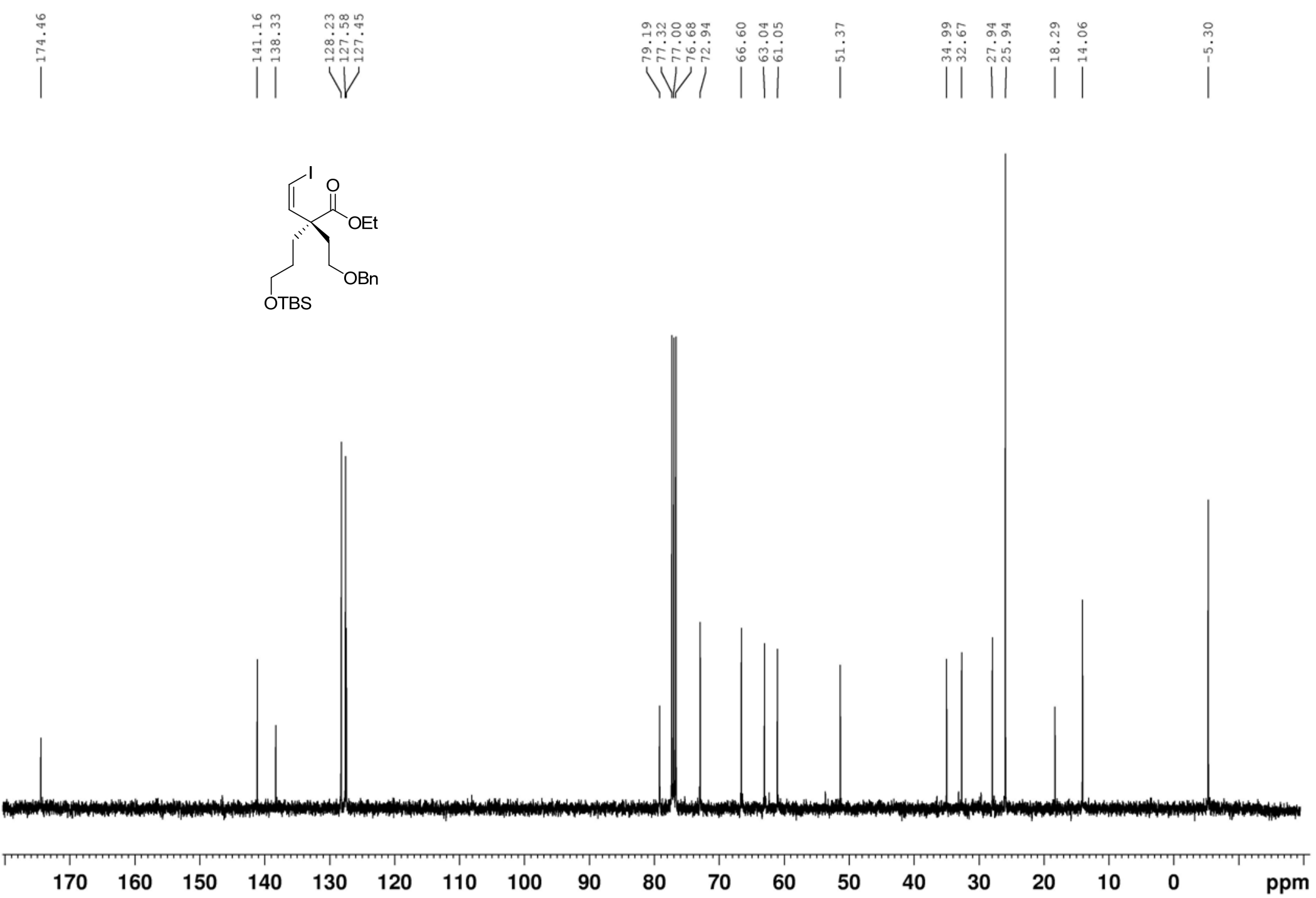


${ }^{1} \mathrm{H}$ NMR of Compound 4 (400 MHz, $\mathrm{CDCl}_{3}$ )

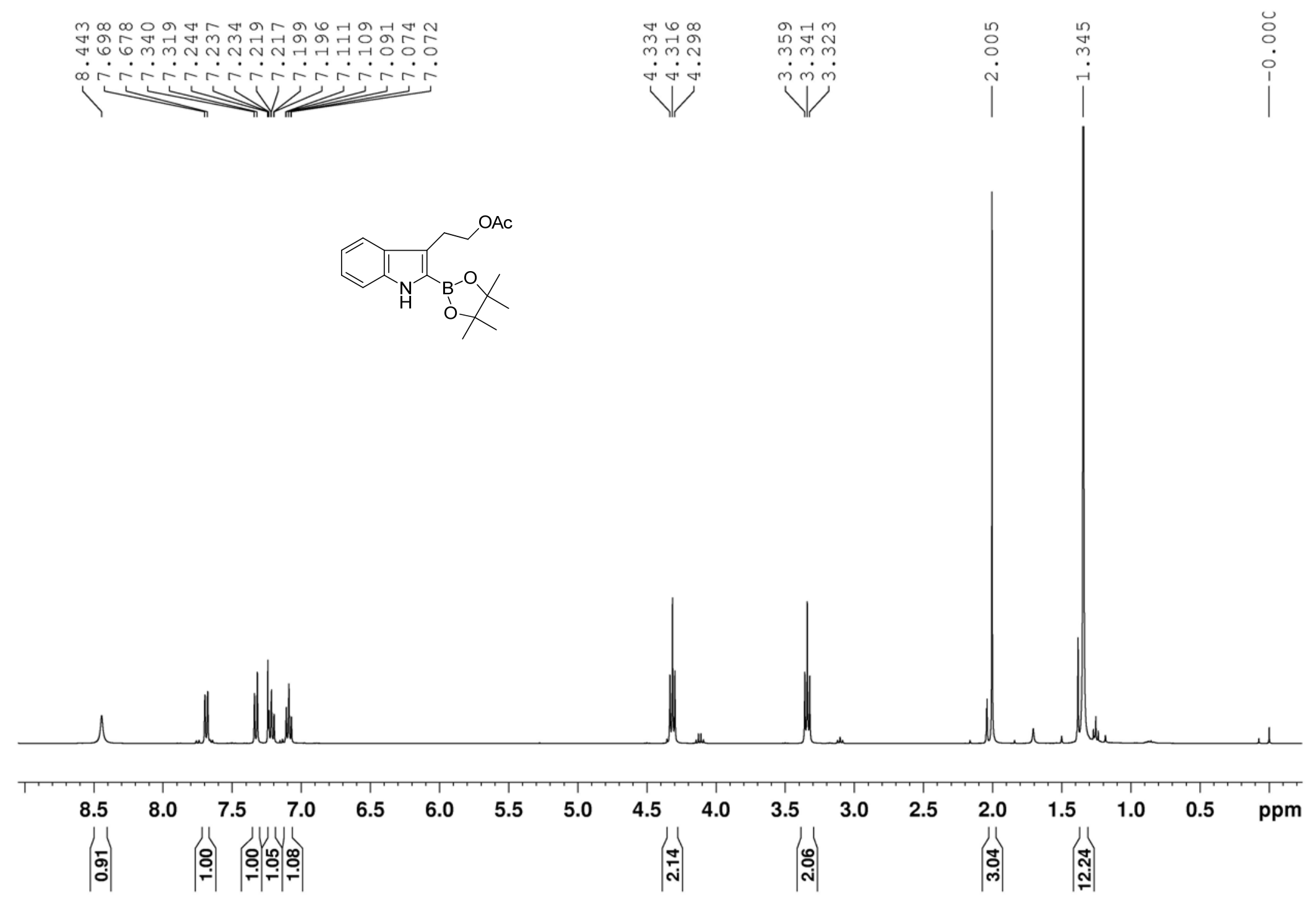


${ }^{13} \mathrm{C}$ NMR of Compound 4 (100 MHz, $\mathrm{CDCl}_{3}$ )

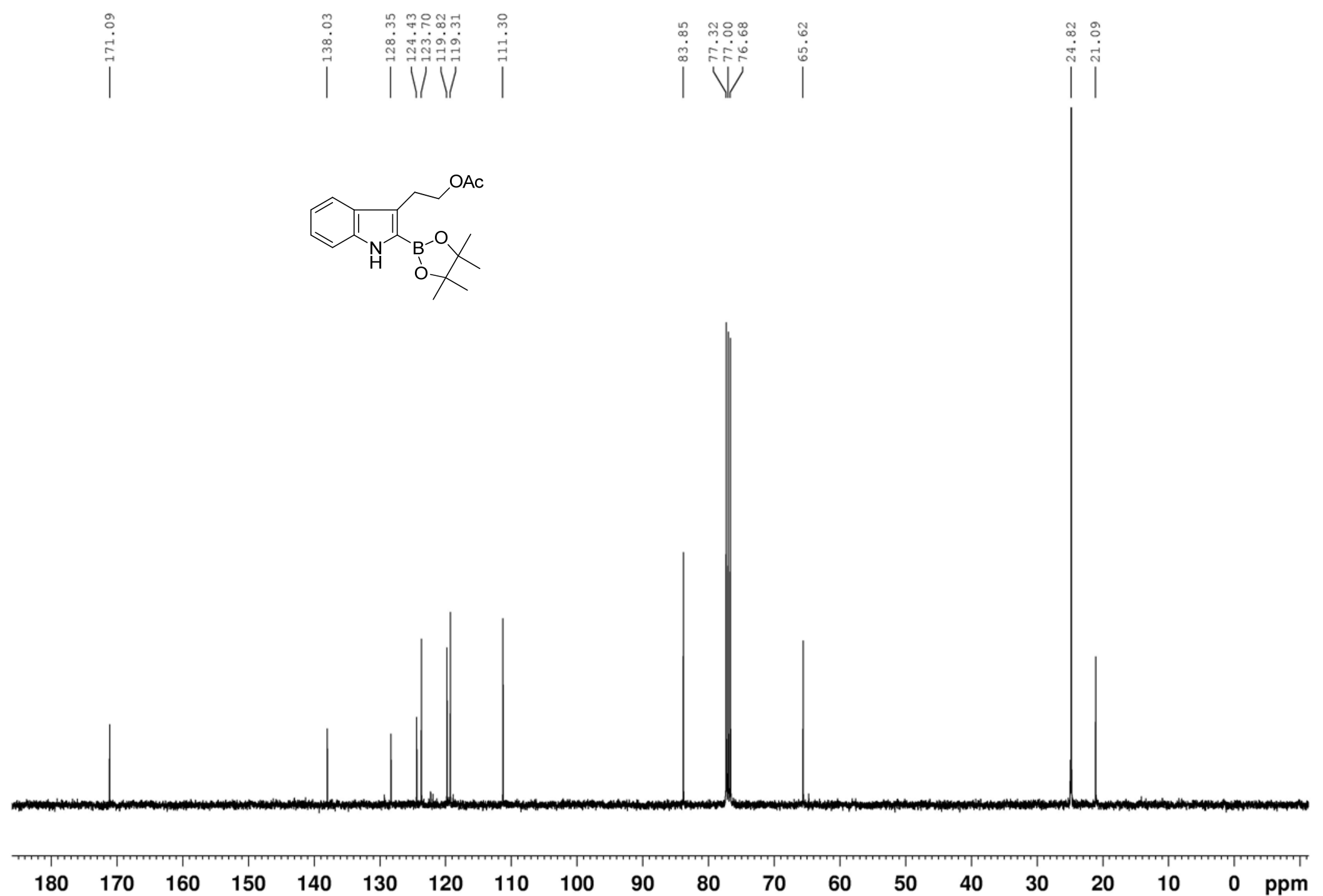


${ }^{1} \mathrm{H}$ NMR of Compound $15\left(400 \mathrm{MHz}, \mathrm{CDCl}_{3}\right)$

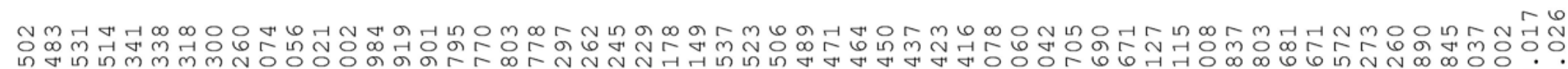
$\underbrace{\infty}_{\infty}$
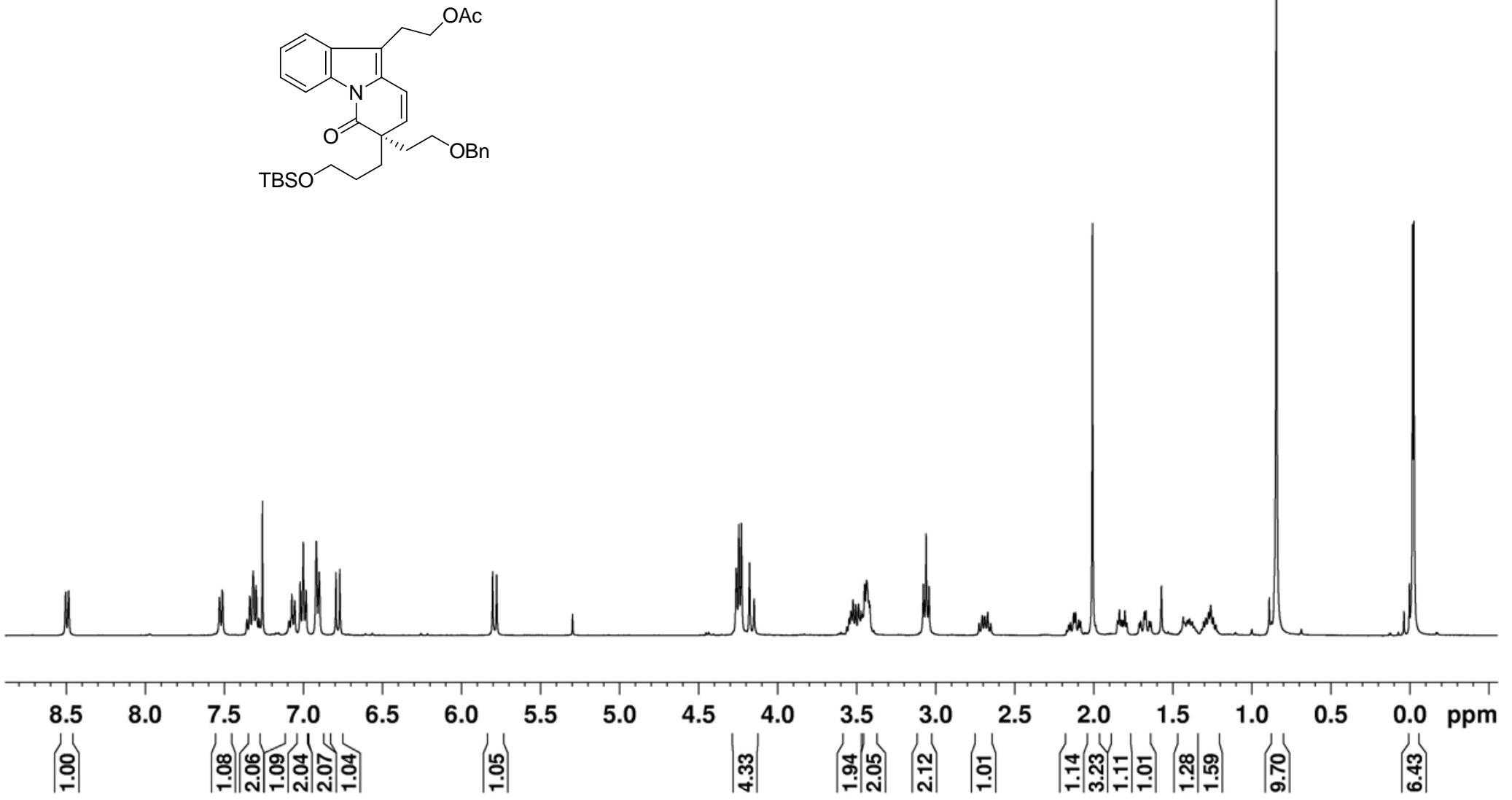
${ }^{13} \mathrm{C}$ NMR of Compound 15 (100 MHz, $\mathrm{CDCl}_{3}$ )

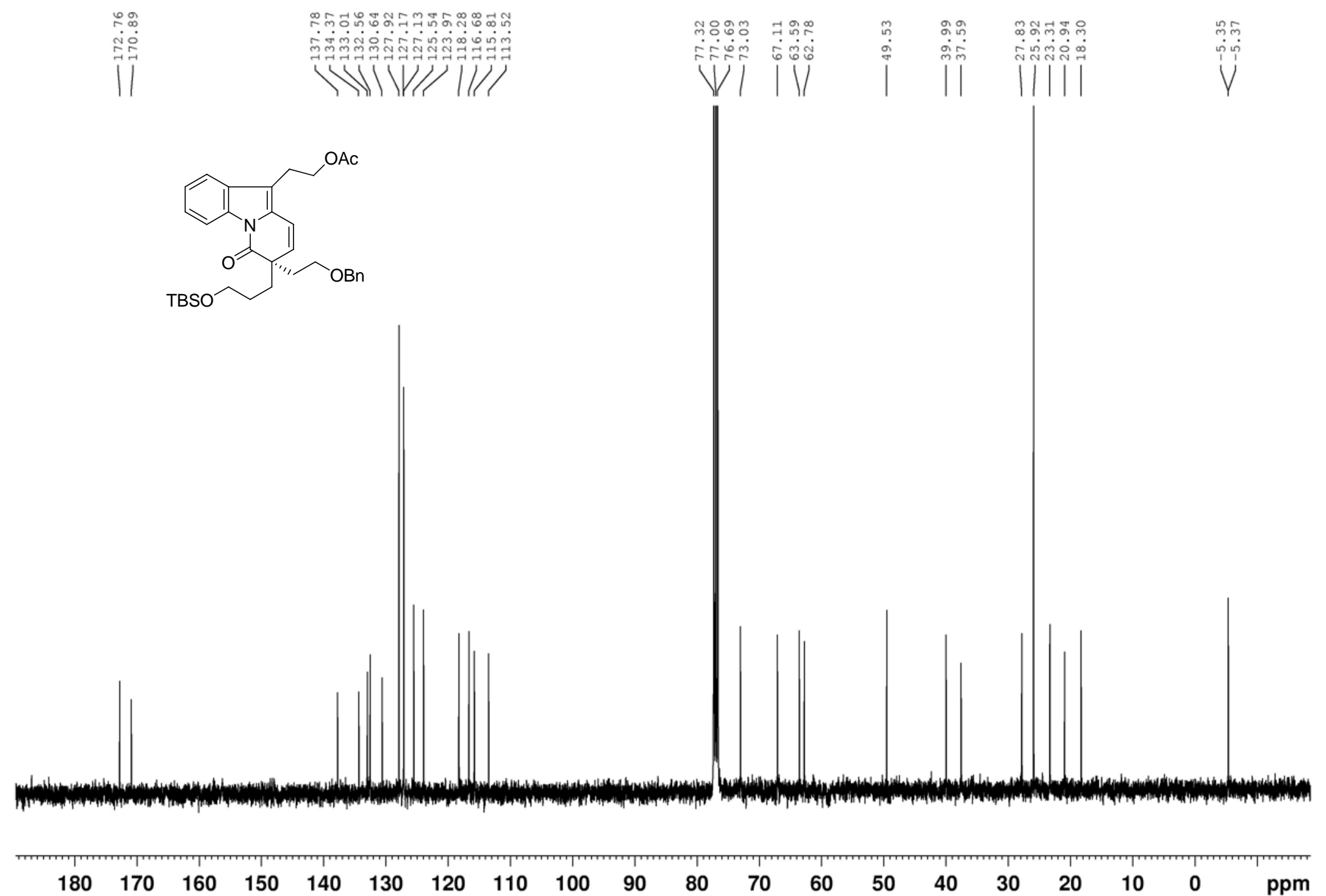


${ }^{1} \mathrm{H}$ NMR of Compound $16\left(400 \mathrm{MHz}, \mathrm{CDCl}_{3}\right)$

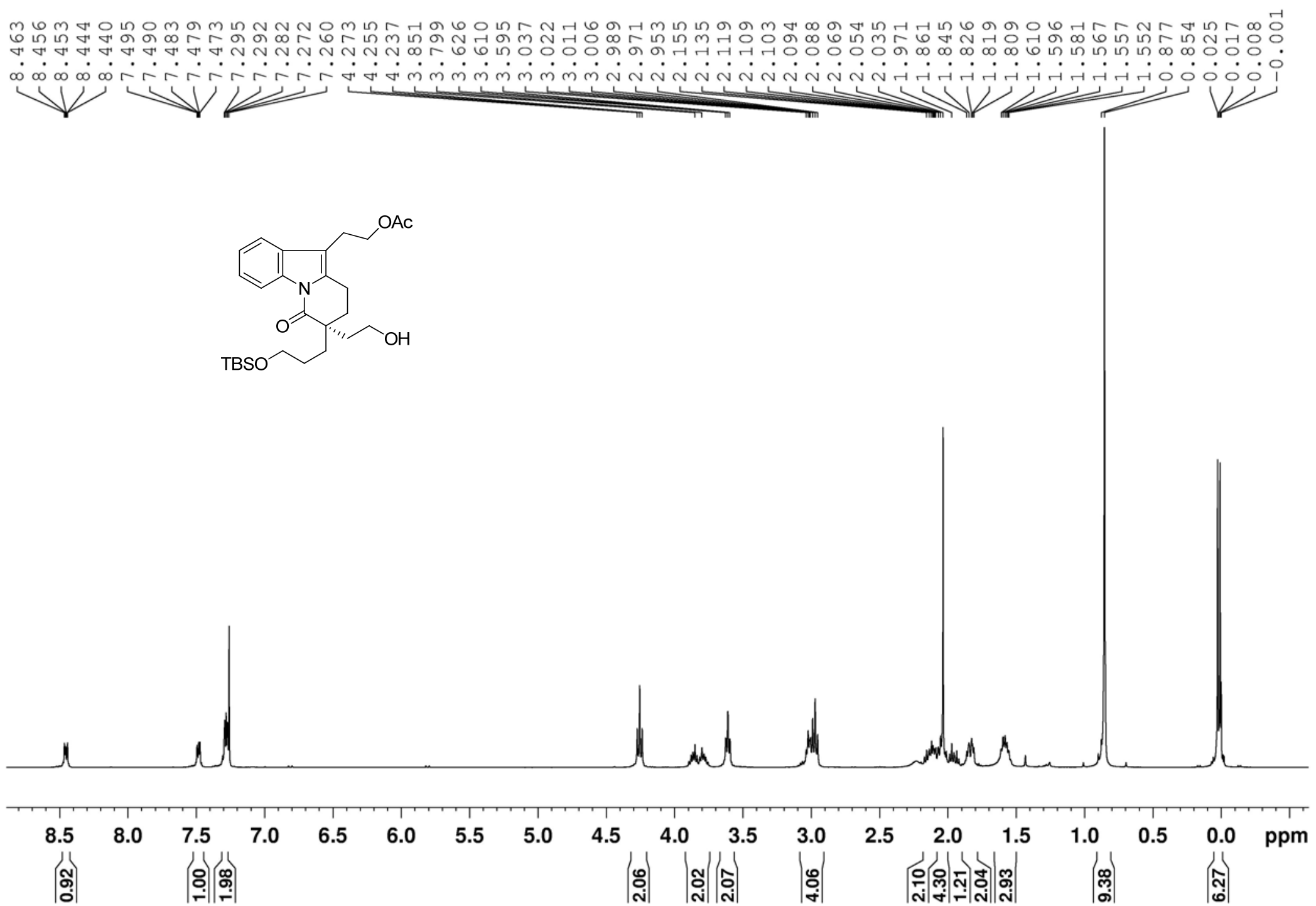


${ }^{13} \mathrm{C}$ NMR of Compound $16\left(100 \mathrm{MHz}, \mathrm{CDCl}_{3}\right)$

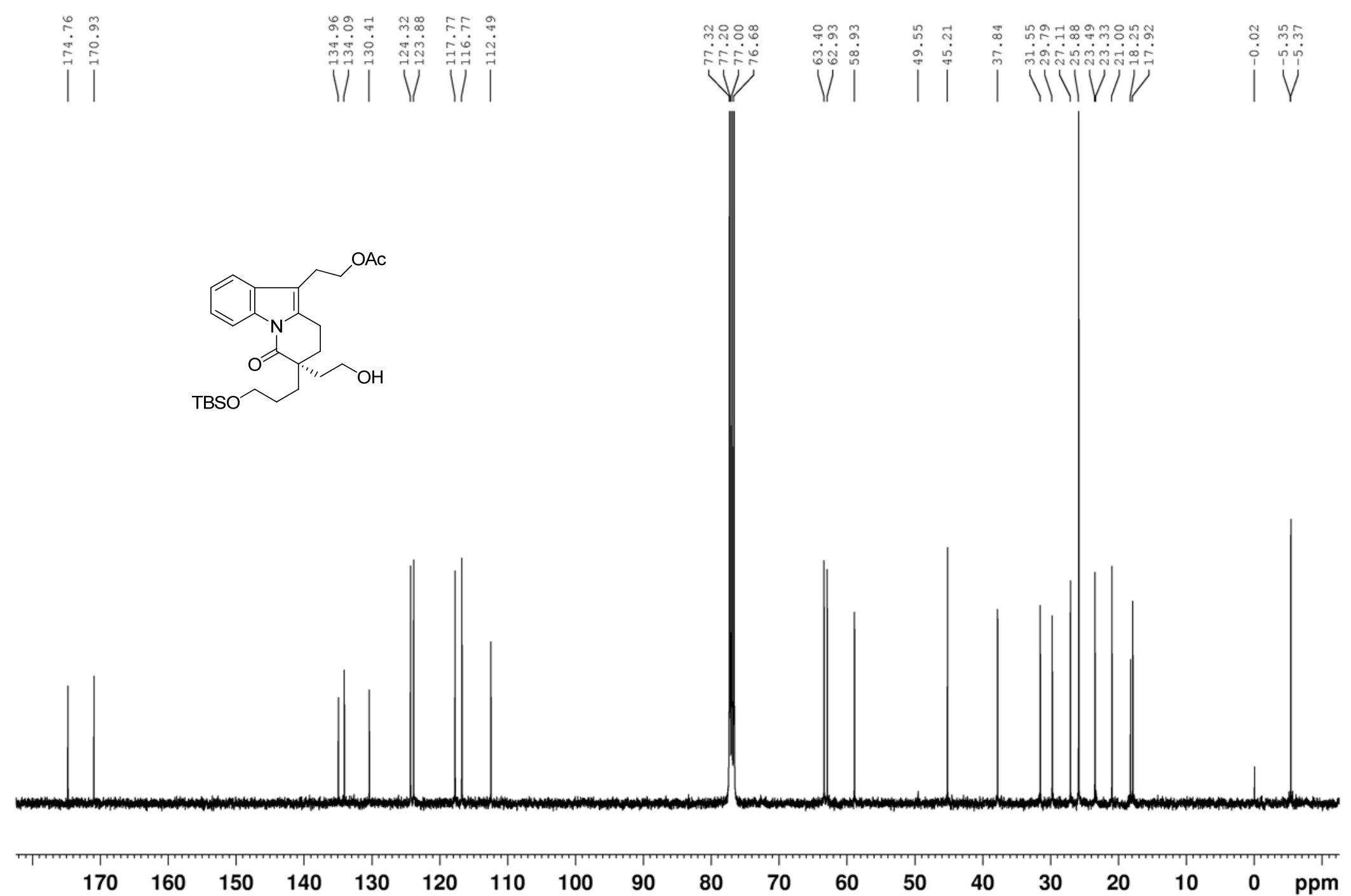


${ }^{1} \mathrm{H}$ NMR of Compound $17\left(400 \mathrm{MHz}, \mathrm{CDCl}_{3}\right)$

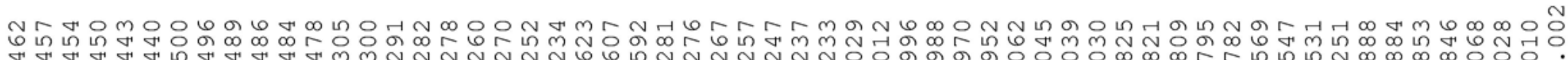

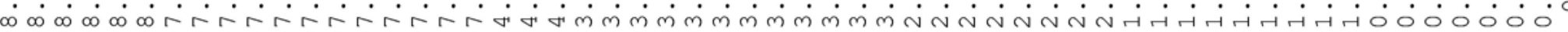
12

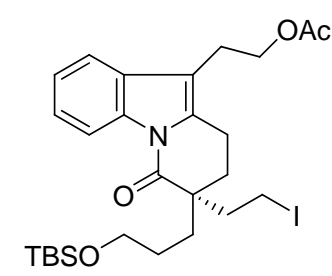

TBSO

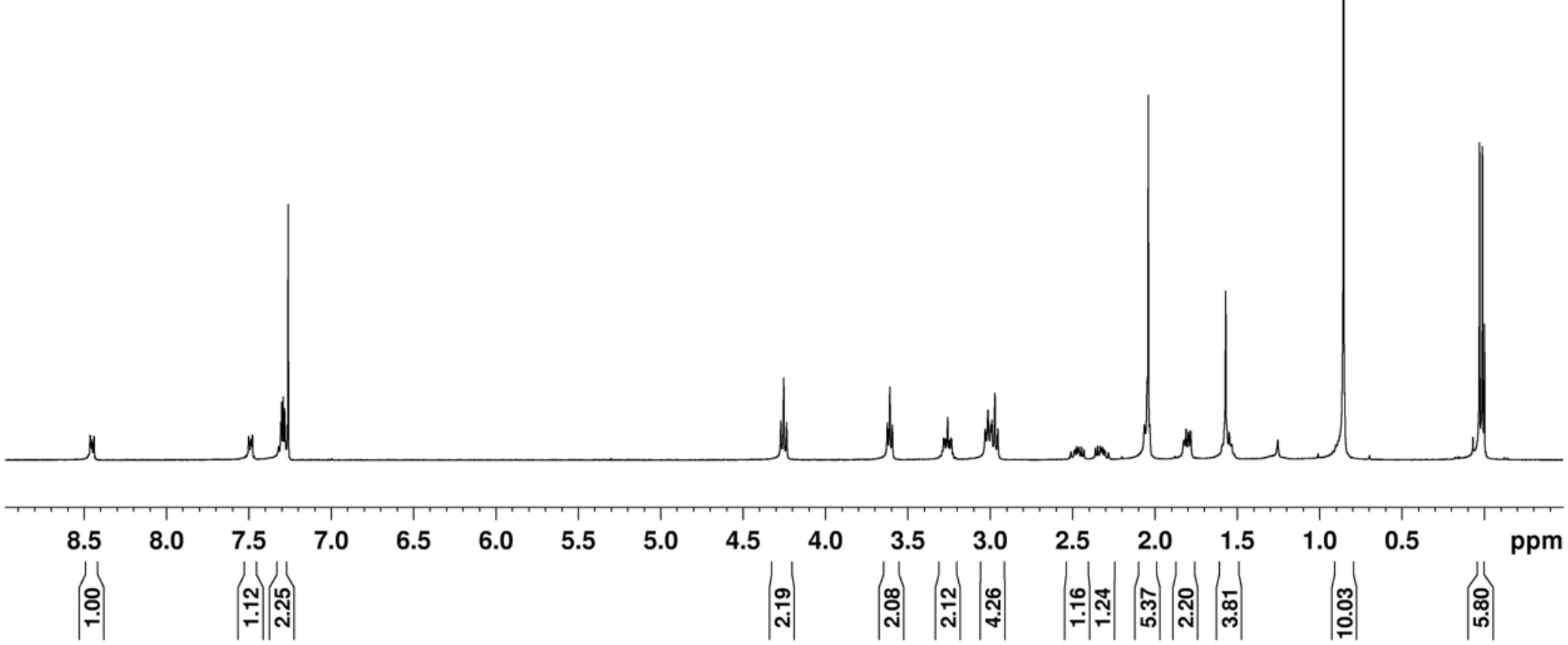


${ }^{13} \mathrm{C}$ NMR of Compound 17 (100 MHz, $\left.\mathrm{CDCl}_{3}\right)$

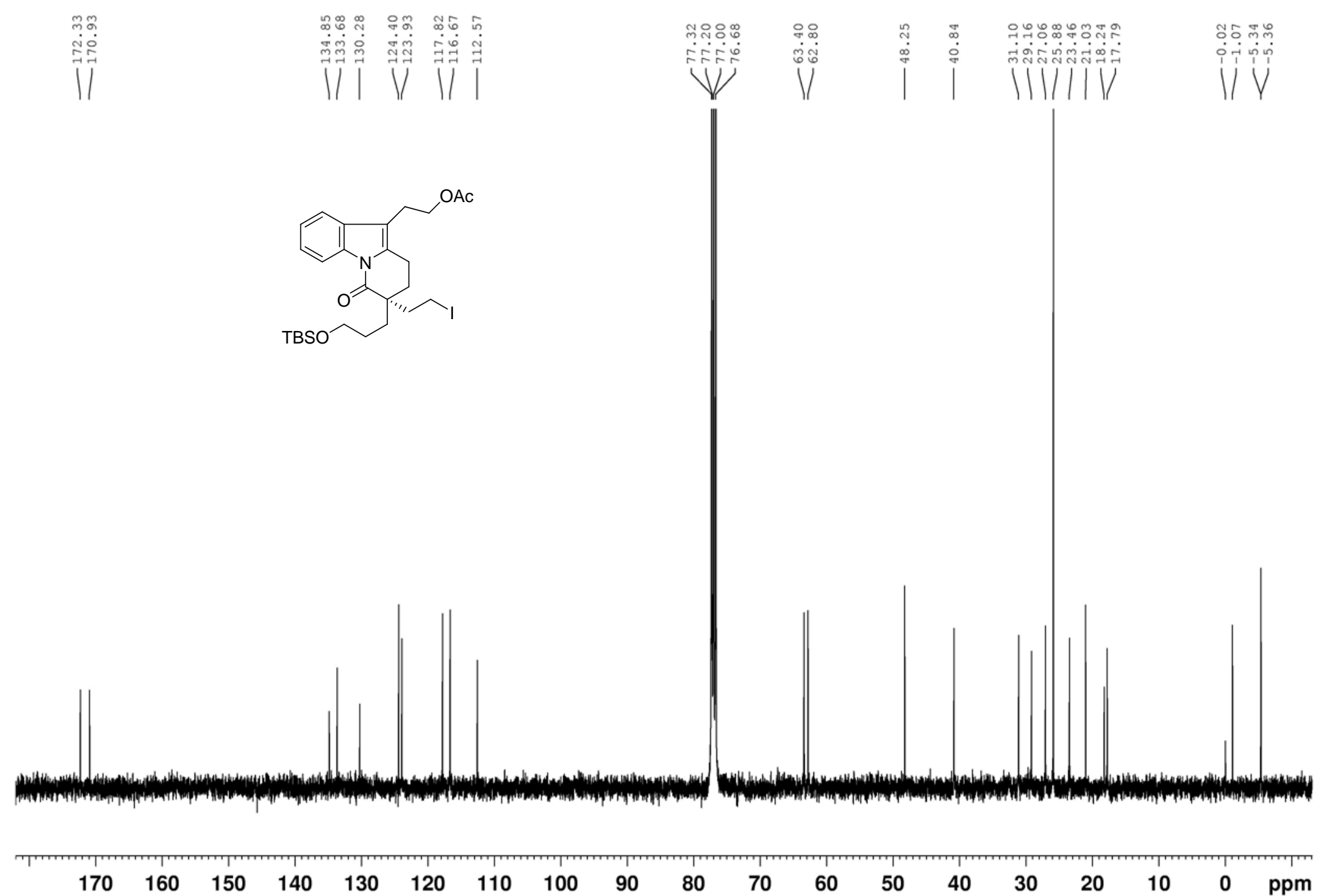




\section{${ }^{1} \mathrm{H}$ NMR of Compound $18\left(400 \mathrm{MHz}, \mathrm{CDCl}_{3}\right)$}

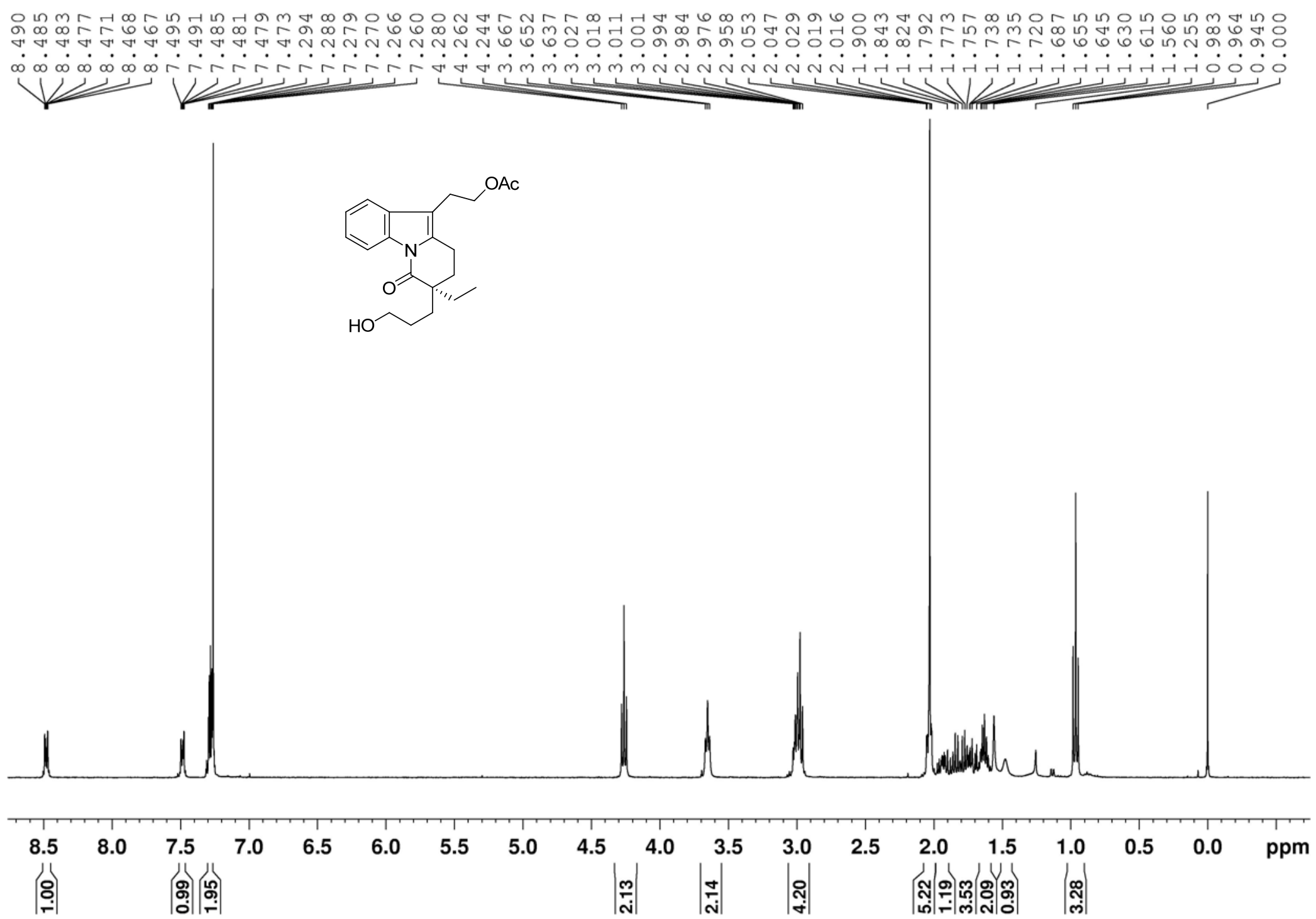


${ }^{13} \mathrm{C}$ NMR of Compound $18\left(100 \mathrm{MHz}, \mathrm{CDCl}_{3}\right)$

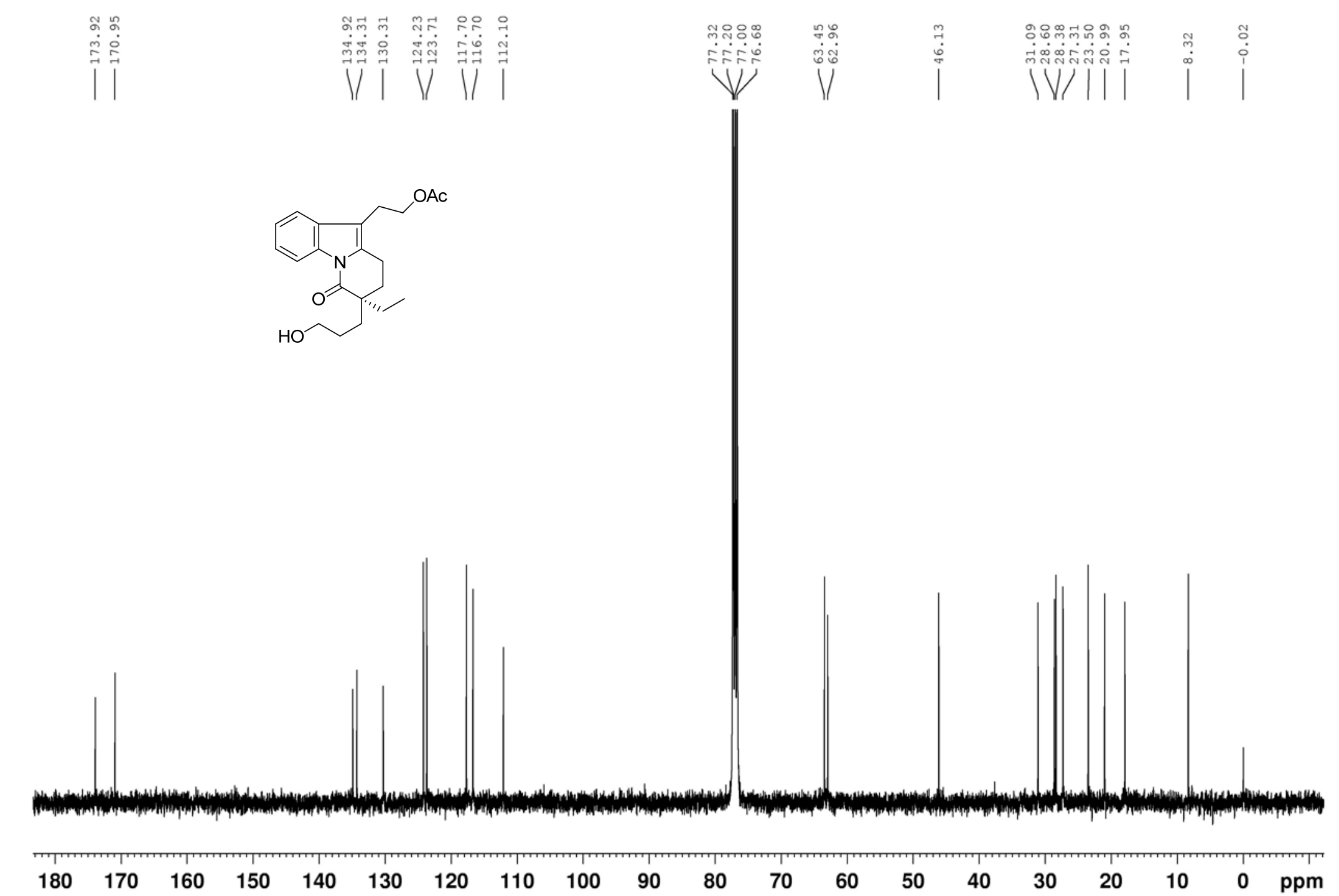


${ }^{1} \mathrm{H}$ NMR of Compound $19\left(400 \mathrm{MHz}, \mathrm{CDCl}_{3}\right)$

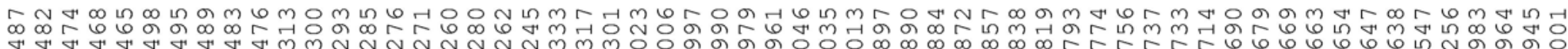

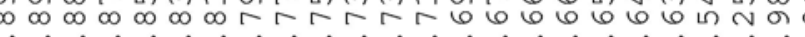
N
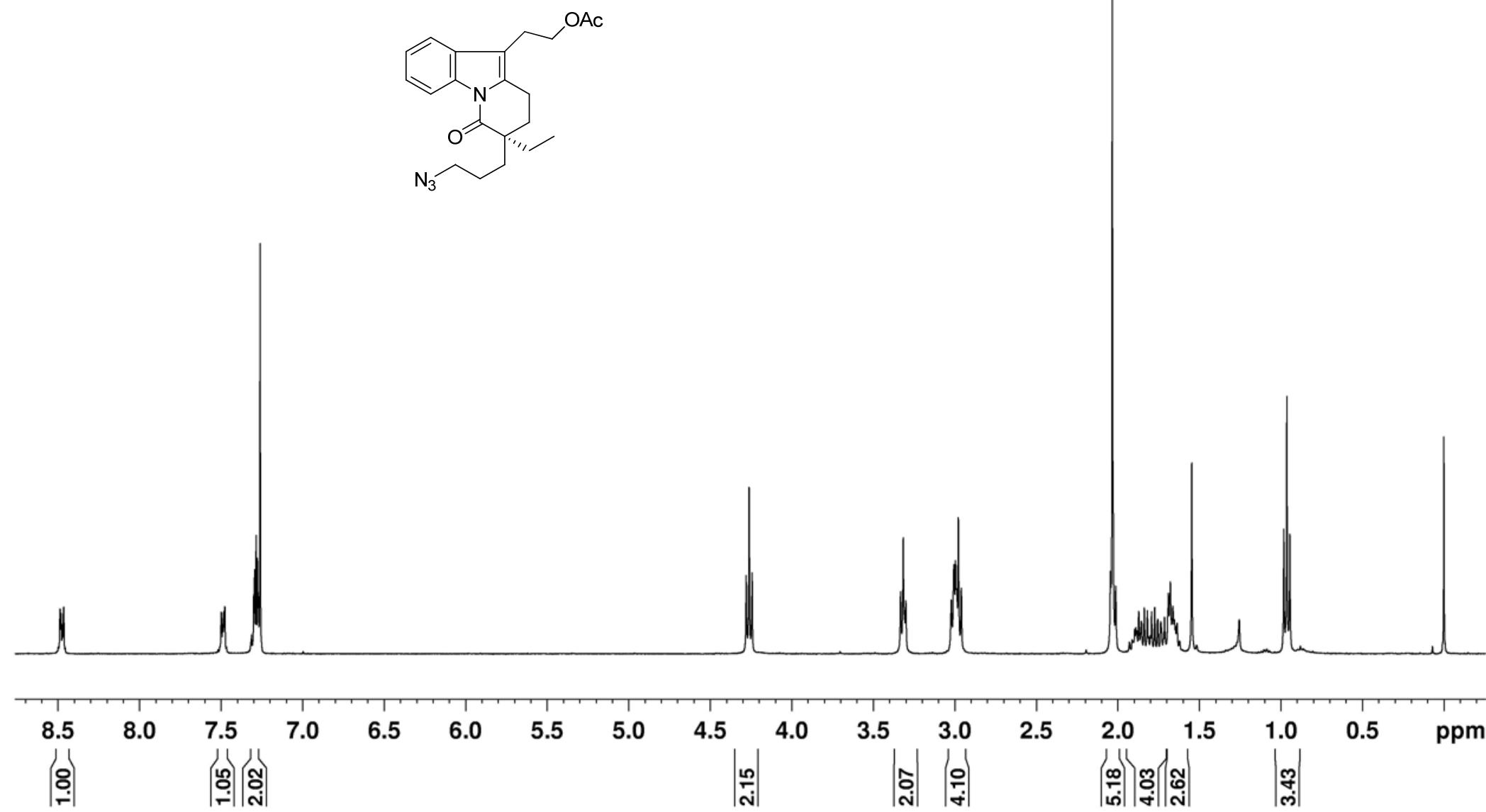
${ }^{13} \mathrm{C}$ NMR of Compound 19 (100 MHz, $\left.\mathrm{CDCl}_{3}\right)$

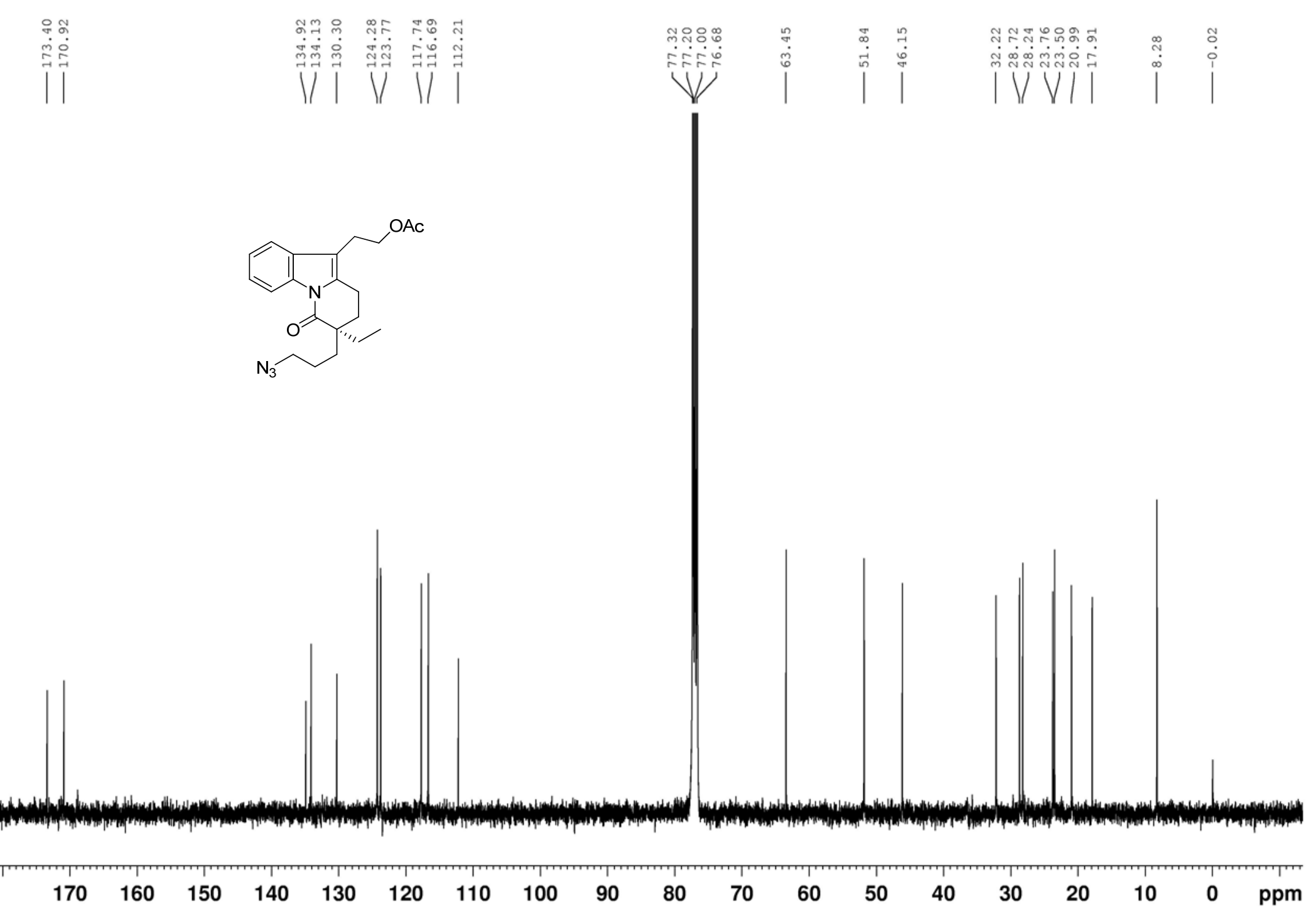


${ }^{1} \mathrm{H}$ NMR of (-)-Goniomitine (400 MHz, $\left.\mathrm{CDCl}_{3}\right)$

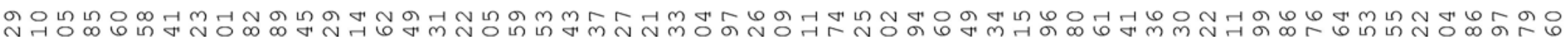

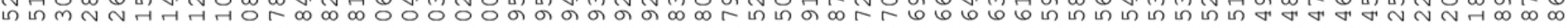

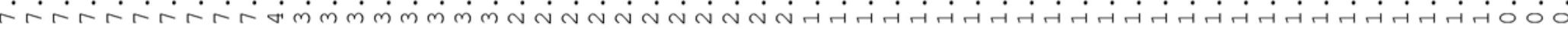

4 l

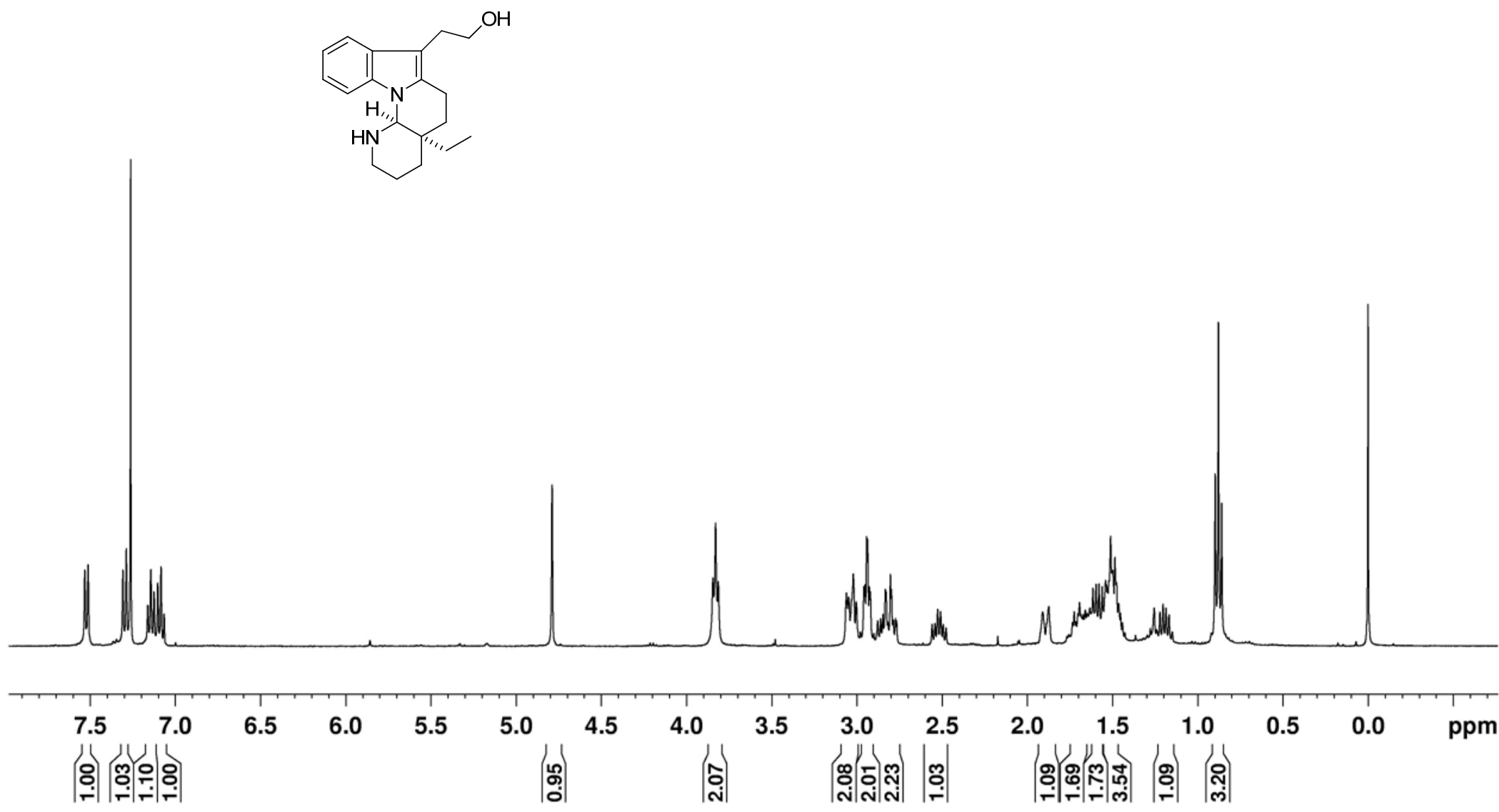


${ }^{1} \mathrm{H}$ NMR of (-)-Goniomitine (400 $\mathrm{MHz}, \mathrm{CDCl}_{3}$ )
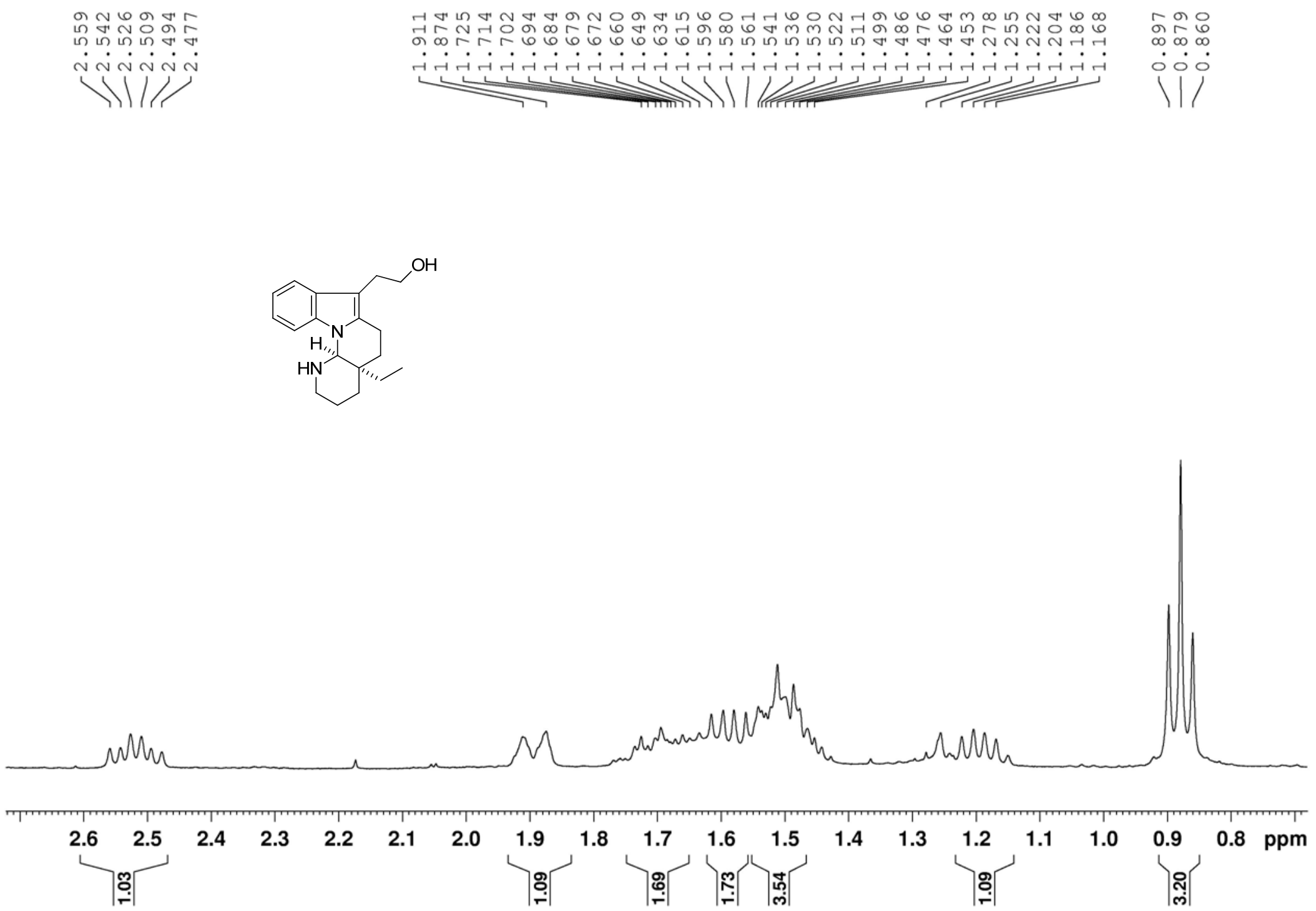
${ }^{13} \mathrm{C}$ NMR of (-)-Goniomitine (100 MHz, $\left.\mathrm{CDCl}_{3}\right)$

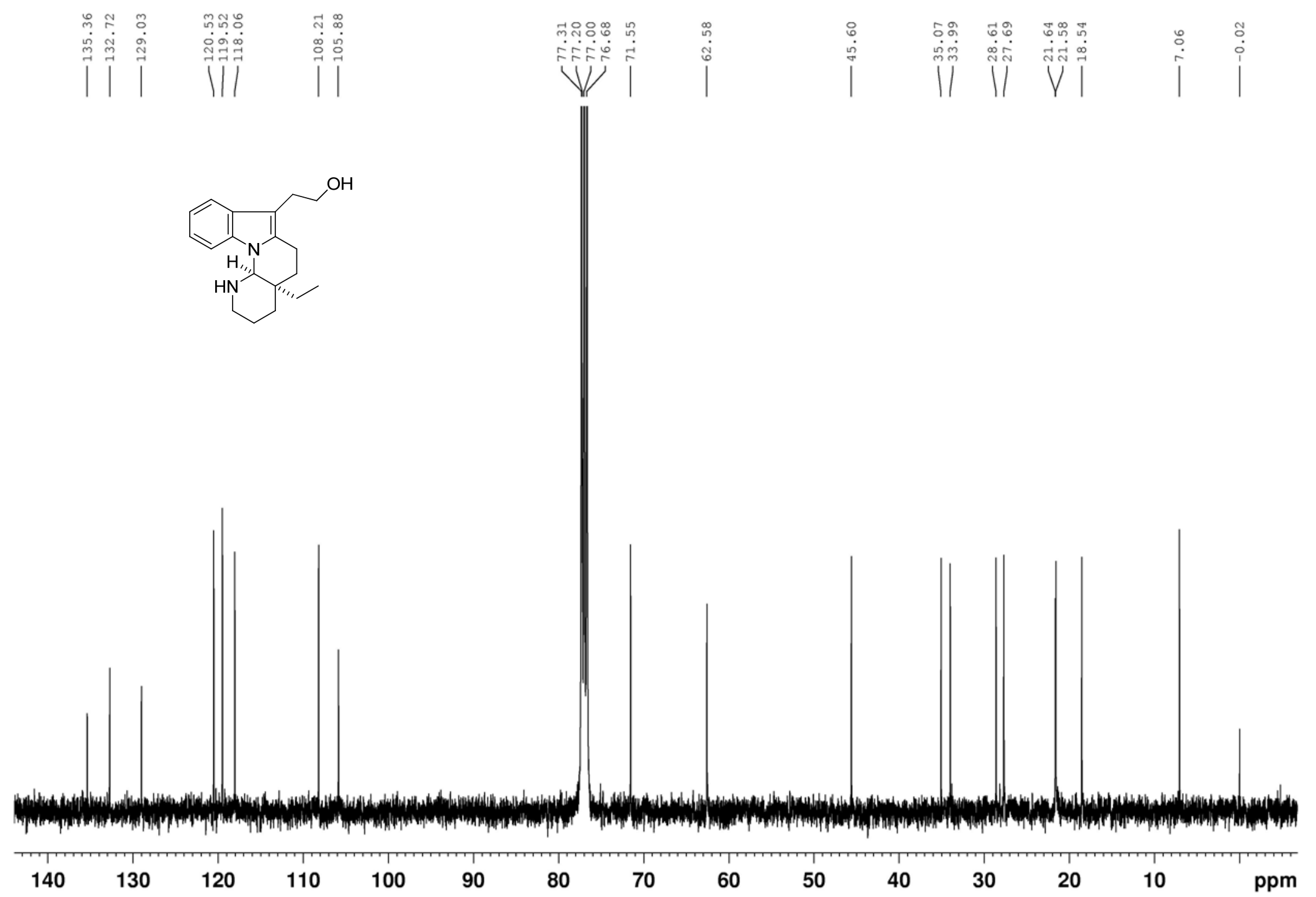

